\title{
Implementing Support for Pointers to Private Data in a General-Purpose Secure Multi-Party Compiler
}

\author{
YIHUA ZHANG, University of Notre Dame \\ MARINA BLANTON, University at Buffalo (SUNY) \\ GHADA ALMASHAQBEH, Columbia University
}

\begin{abstract}
Recent compilers allow a general-purpose program (written in a conventional programming language) that handles private data to be translated into a secure distributed implementation of the corresponding functionality. The resulting program is then guaranteed to provably protect private data using secure multi-party computation techniques. The goals of such compilers are generality, usability, and efficiency, but the complete set of features of a modern programming language has not been supported to date by the existing compilers. In particular, recent compilers PICCO and the two-party ANSI C compiler strive to translate any C program into its secure multi-party implementation, but they currently lack support for pointers and dynamic memory allocation, which are important components of many C programs. In this work, we mitigate the limitation and add support for pointers to private data and consequently dynamic memory allocation to the PICCO compiler, enabling it to handle a more diverse set of programs over private data. Because doing so opens up a new design space, we investigate the use of pointers to private data (with known as well as private locations stored in them) in programs and report our findings. Aside from dynamic memory allocation, we examine other important topics associated with common pointer use such as reference by pointer/address, casting, and building various data structures in the context of secure multi-party computation. This results in enabling the compiler to automatically translate a user program that uses pointers to private data into its distributed implementation that provably protects private data throughout the computation. We empirically evaluate the constructions and report on the performance of representative programs.
\end{abstract}

CCS Concepts: • Security and privacy $\rightarrow$ Privacy-preserving protocols; $\bullet$ Software and its engineering $\rightarrow$ Software creation and management;

Additional Key Words and Phrases: Secure multi-party computation, pointers to private data, dynamic memory management, $\mathrm{C}$ compiler, secret sharing, oblivious data structures

\section{ACM Reference format:}

Yihua Zhang, Marina Blanton, and Ghada Almashaqbeh. 2017. Implementing Support for Pointers to Private Data in a General-Purpose Secure Multi-Party Compiler. ACM Trans. Priv. Secur. 21, 2, Article 6 (December 2017), 34 pages.

https://doi.org/10.1145/3154600

\footnotetext{
This work was supported in part by Grants No. CNS-1319090 and No. CNS-1223699 from the National Science Foundation and FA9550-13-1-0066 from the Air Force Office of Scientific Research. Any opinions, findings, and conclusions or recommendations expressed in this publication are those of the authors and do not necessarily reflect the views of the funding agencies.

Authors' addresses: Y. Zhang, Department of Computer Science and Engineering, University of Notre Dame, Notre Dame, IN 46556, USA; email: yihua1023@gmail.com; M. Blanton, Department of Computer Science and Engineering, University at Buffalo, Buffalo, NY 14260, USA; email: mblanton@buffalo.edu; G. Almashaqbeh, Department of Computer Science, Columbia University, New York, NY 10027, USA; email: ghada@cs.columbia.edu.

Permission to make digital or hard copies of all or part of this work for personal or classroom use is granted without fee provided that copies are not made or distributed for profit or commercial advantage and that copies bear this notice and the full citation on the first page. Copyrights for components of this work owned by others than ACM must be honored. Abstracting with credit is permitted. To copy otherwise, or republish, to post on servers or to redistribute to lists, requires prior specific permission and/or a fee. Request permissions from permissions@acm.org.

(c) 2017 ACM 2471-2566/2017/12-ART6 \$15.00
}

https://doi.org/10.1145/3154600

ACM Transactions on Privacy and Security, Vol. 21, No. 2, Article 6. Publication date: December 2017. 


\section{INTRODUCTION}

Recent advances in secure multi-party computation make it feasible to securely compute with private data belonging to different organizations even for complex functionalities. Furthermore, together with ubiquitous proliferation of cloud computing services, these techniques give rise to secure computation outsourcing. For these reasons, the research community has recently developed a number of compilers for transforming a general-purpose program into its secure distributed implementation (see, e.g., References [10,26]). These tools aim at generality and are designed to translate a program written in a conventional programming language into an equivalent program that uses secure computation techniques to protect private data. They also aid usability and make it easier for a programmer without extensive knowledge of secure computation techniques to produce a protocol that can be securely executed in a distributed environment.

It has been long known that any computable function can be securely evaluated by multiple participants if it is represented as an arithmetic or Boolean circuit. This representation, however, is not always obvious or known, and representing a program as a circuit may significantly increase the program size. Existing compilers remove the need for the programmer to perform this translation manually and assemble secure implementations from efficient building blocks for elementary operations. Thus, efficiency of the resulting secure computation is also one of the goals that compilers target. Furthermore, the ability to support both private (i.e., protected) and public (i.e., not protected) data or variables in a single program adds a level of complexity to the implementation because of the need to support interactions between public and private variables and secure data flow enforcement.

While the design goal of several compilers was to support any feature of a general-purpose programming language (such as C in References [10] and [26]), all such compilers have limitations. In particular, the original version of the PICCO compiler [26] provided no direct support for C pointers (i.e., pointers were supported only in the form of static arrays) and, as a result, no support for dynamic memory allocation other than static arrays. Similarly, the original version of the twoparty compiler for ANSI C [10] supported pointers only in the form of statically allocated arrays restricted to a constant size and had additional limitations (such as support for floating point arithmetic was not available in the open source CBMC that the compiler builds upon). Thus, support for C-like pointers-or, in other programming languages, support for the features that pointers enable such as dynamic memory allocation, reference by pointer or address, and building data structures-is the most crucial part of a general-purpose program that is currently unavailable in existing compilers. Adding this support is thus the focus of our work.

In this article, we put forward a framework for supporting pointers to private data and consequently realize it in the PICCO compiler [26]. $\mathrm{PICCO}^{1}$ is a source-to-source translator that takes as an input a $\mathrm{C}$ program with variables to be protected marked as private and produces a $\mathrm{C}$ program that implements the computation using secure multi-party computation techniques based on linear secret sharing. We view PICCO as an attractive choice of a C compiler because of the flexibility of the setting it uses. In particular, the setting assumes three groups of participants: (i) input parties who hold private inputs into the computation, (ii) computational parties who perform secure computation on secret-shared data, and (iii) output parties who are entitled to learn the result of the computation. The composition of these three groups can be arbitrary (in particular, including the same, overlapping, or non-overlapping groups), which makes the setting suitable for secure multi-party computation (SMC), delegation of the computation by multiple data owners to a subset of them or other suitable entities, or secure computation outsourcing by one or more parties. This

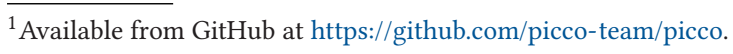


flexibility follows from the use of secret sharing techniques and may or may not be present in tools that build on alternative secure computation techniques (such as, e.g., garbled circuit evaluation).

With linear secret sharing, before secure computation can commence, each input party splits her private inputs into $n>2$ secret shares, where $n$ is the number of computational parties, and communicates each share to the respective computational party. The computational parties then proceed with evaluating the function on secret-shared data and communicate their shares of the result to the output parties who reconstruct the output using their shares. Any linear combination of secret-shared integers is performed locally, but multiplication of secret-shared integers constitutes an elementary interactive operation. Performance is then measured in the total number of interactive operations as well as the number of sequential interactions or rounds, and recent solutions based on secret sharing aim at minimizing overhead using both metrics.

When PICCO is used to perform source-to-source translation, the input program is a conventional C program where each variable is marked to be either private or public. All computation with private variables is transformed into secure arithmetic on shared data, while operations with public variables that do not interact with private data are left unchanged. In addition to specifying private/public qualifies for each variable, for performance reasons PICCO also allows the programmer to mark the places where computation can proceed concurrently (i.e., to decrease the number of computation rounds), which also extends the conventional $\mathrm{C}$ syntax.

Adding pointer support to a program that manipulates private data not only extends the compiler to handle the full range of $\mathrm{C}$ programs (that do not violate secrecy of private data), but also permits important features of programming languages, treatment of which, to the best of our knowledge, has not been done before. As part of this work, we thus explore how pointers to private data (including pointers with private locations) can be implemented and discuss our design decisions. We also study common uses of pointers in programs and the impact our implementation has on those language features. For example, enabling dynamic memory allocation is an important aspect of this work. Based on our analysis as well as empirical evaluation, several of these features introduce only marginal costs. Also, one of the important topics studied in this work is the use of pointer-based data structures written for private data. Our results indicate that the use of pointers (to private data) is very attractive and maintains high efficiency for several popular data structures. In some other cases, in particular when working with sorted data, privately manipulating pointers increases complexity of data structure operations and it might be desirable to pursue alternative implementations.

We emphasize that it is not the goal of this article to try to develop most efficient implementations for different data structures. Instead, the goal is to determine how pointers to private data can be supported at a low possible cost and to what performance of typical programs that might lead. We note that, depending on the program structure, asymptotic complexity of a translated program might be higher than that of the original. For example, consider an if-then-else statement with a private condition (e.g., conditional statements used in traversing a binary tree). When data privacy is not required, only one of the two branches will be executed, but with any compiler that produces a secure implementation both branches will have to be evaluated to hide the result of the private condition. Then, with a sequence of $n$ nested if-then-else statements, in the worst case the secure program might have to execute $O\left(2^{n}\right)$ instructions where the original program would execute only $O(n)$. This means that the general translation approach can lead to an exponential increase in the runtime for programs of practical relevance. As part of this work, we show that data structures that utilize pointers to private data cover the entire spectrum of possibilities: in one extreme, they result in no asymptotic increase over conventional non-secure counterparts, and in another extreme, the increase is exponential. This provides insights on when natural pointer use is very attractive and when other, alternative implementations might be desired. 
While for many data structures alternative, non-pointer-based implementations may be possible, we note that our extension of PICCO with pointers enables support for an important aspect of modern programs otherwise not available in any secure multi-party compiler we are aware of, which is dynamic memory allocation. Dynamic memory allocation is essential for a generalpurpose programming language, but has not been systematically studied in the context of secure multi-party computation (e.g., even publications and compilers that run secure computation on data sets of a large size assume that the size of the data is fixed and known at compilation time). As an example, consider an application in which data items arrive over time. A pointer-based linked list will allow for a natural and graceful mechanism of memory management, that unlike statically allocated arrays does not require complex provisions for allocating a larger buffer when the current one becomes full, moving the data, or merging previously allocated buffers.

The rest of this article is organized as follows: We first give a brief overview of related work in Section 2. After presenting background information in Section 3, we proceed with generically presenting our solution for supporting pointers to private data in Section 4. In Section 5, we discuss dynamic memory management, while Section 6 addresses specifics of pointer implementation in PICCO. Section 7 next summarizes operations with pointers to private data and formally shows security of the design. Section 8 analyzes various data structures built using pointers to private data. Last, Section 9 presents the results of performance evaluation of representative programs that utilize pointers (to private data) and Section 10 concludes this work.

\section{RELATED WORK}

In this section, we review the most closely related work on SMC compilers and secure/oblivious data structures. Regarding the compilers, Fairplay [16] was a pioneer work that enables compilation of secure two-party protocols based on garbled circuits. Its extension to multiple parties, FairplayMP [3], implements secure computation using Boolean circuits and secret sharing techniques. TASTY [9] is another two-party SMC compiler that combines garbled circuit techniques with those based on homomorphic encryption. Its follow-up ABY [8] provides more efficient mixed-mode two-party computation using Boolean and arithmetic circuits. Sharemind [4] and VIFF [6] are multi-party compilers based on custom additive three-party secret sharing and standard threshold linear secret sharing, respectively. All of the above compilers use custom domain-specific languages to represent user programs. The two-party compiler for ANSI C [10] and PCF [13] both use two-party garbled circuit techniques, where the former's goal is to support general purpose $\mathrm{C}$ programs, while the latter uses a new circuit format and employs optimizations to reduce the compilation time and storage. Last, TinyGarble [21] uses hardware synthesis to optimize garbled circuits for two-party computation and its follow-up [7] also provides optimizations for arithmetic circuits. All of these compilers require linear in the size of memory work to access memory at a private location. SCVM [14], on the other hand, is an automated compiler that utilizes oblivious RAM (ORAM) and targets two-party computation. ObliVM [15] is another ORAM-based secure two-party computation compiler that transforms programs written in high-level abstractions to optimized garbled circuit implementations. Finally, a recent compiler Frigate [18] was designed to guarantee correctness of programs compiled into circuits for secure two-party computation.

To support data structures in the SMC framework, several solutions [11, 17, 22-24] have been proposed. The main motivation of this line of work is the need to store and manipulate private data in an efficient and flexible manner. Toft [22] proposed a private priority queue that has a deterministic access pattern as opposed to randomized ones in ORAM-based data structures. Similarly, Zahur and Evans [24] build static circuits for stacks, queues, and associative maps of complexities substantially lower than touching all data items in the data structure in linear time. On the other hand, Keller and Scholl [11] introduced implementations of arrays, dictionaries, 
and priority queues based on various flavors of ORAM implementations. Mitchell and Zimmerman [17] also provide implementations of stacks, queues, and priority queues based on oblivious data compaction and an offline variant of ORAM. Wang et al. [23] proposed implementations of maps, sets, priority queues, stacks, and deques based on ORAM techniques modified for specific data access patterns. Different from all of the above, our work provides support for dynamic data structures in a generic way as found in general purpose programming languages. That is, the programmer has the basic tools and primitives that enable her to build any desired data structure.

In our implementation, a pointer to private data may store one or more locations where the data might reside, which in the worst case is linear in the program's memory size. ORAM-based techniques, on the other hand, guarantee that when an item is accessed at a private location, the number of accessed memory locations is polylogarithmic in the total memory size. Thus, our general solution may or may not be faster than using ORAM, depending on both the program and the data size. As we discuss later in this work, employing ORAM techniques can be beneficial for certain data structures (and sufficiently large data sets). Building custom data structures, however, is beyond the scope of this work.

One of the applications that the compiler can naturally be used for once support for pointers to private data is in place is evaluation of a context-free grammar on private data (implemented as a shift-reduce parser using a stack). The grammar can be either public or private, and in the latter case execution will correspond to evaluation of private expressions/programs on private data. Techniques for evaluation of private programs (on private data) are a separate area of research, discussion of which is beyond the scope of this work, but the reader may refer to recent results in this areas such as those in References [12,20].

\section{BACKGROUND INFORMATION}

PICCO uses Shamir secret sharing [19] for implementing secure arithmetic and other operations on private data. It is an $(n, t)$-threshold linear secret sharing scheme, in which a private value is represented using $n>2$ secret shares, one held by each computational party. Then, any $t+1$ or more shares can be used to reconstruct the private value, while $t$ or fewer parties cannot learn any information about the shared value (which is perfectly protected in the information-theoretic sense). In a linear secret-sharing scheme, a linear combination of secret-shared values can be performed by each computational party locally, without any interaction, while multiplication of secret-shared values requires communication between all of them. With Shamir secret sharing, computation takes place over a field of a desired size (larger than any value that needs to be represented). A secret $s$ is represented using a random polynomial of degree $t$ with the free coefficient set to $s$, and each share corresponds to the evaluation of the polynomial on a distinct non-zero point. Given $t+1$ or more shares, the secret can be reconstructed using Lagrange interpolation. Then, addition or subtraction of secret-shared values, or multiplication of a secret-shared value by a known integer can be performed by each party locally using its shares. Multiplication involves multiplying two shares, which raises the corresponding polynomial degree to $2 t$, and resharing and interpolating the result to bring the degree of the corresponding polynomial from $2 t$ to $t$. This imposes the requirement that $t<n / 2$. With the way multiplication is performed, it is also possible to evaluate any multi-variate polynomial of degree 2 over secret-shared integers with a single interaction. That is, we first evaluate the polynomial and re-share the overall result instead of doing so for the intermediate products. This serves as a powerful optimization tool when, for instance, the dot product of two secret shared vectors/arrays needs to be computed, which results only in a single interaction.

While the regular field operations achieve perfect secrecy, implementation of some of the basic operations used in PICCO (such as comparisons, division, etc.) is statistically secure, which 
requires the bitlength of the field elements to be increased by the statistical security parameter. This slightly increases the cost of field operations, as well as the amount of communication associated with transmitting field elements. The optimal size of field elements is automatically determined by PICCO for each program it compiles.

Performance of these techniques is measured in terms of the total number of elementary interactive operations (field multiplications or reconstructions of a value from its shares) as well as the number of rounds. For that reason, PICCO supports a number of optimizations to reduce the round complexity of programs that it outputs through concurrent or batch execution.

Support for pointers to private data (and the corresponding functionalities such as dynamic memory management) was the only missing functionality in PICCO. Thus, we modify the compiler to enable it to compile user programs that contain pointers to private data, which was not previously available. Our changes to the compiler affect only pointers to private data and the introduction of two built-in functions for memory allocation and deallocation (called pmalloc and pfree) associated with pointers to private data.

\section{ADDING POINTER SUPPORT}

Recall that in $\mathrm{C}$ a pointer is a variable of a special type that stores a location in memory at which data of a particular type can be located. Because a pointer stores an address, it can be treated very generally with the possibility of directly manipulating pointers, changing the addresses they store, dereferencing a pointer to access the data to which it points, casting a pointer of a particular type to a pointer of another type, and using pointers to functions. As with any SMC compiler, our security objective is to provably reveal no information about private data used in the computation.

\subsection{Working Toward a Solution}

When working with pointers in the presence of private data, besides traditional $\mathrm{C}$ pointers to public variables, we can distinguish between pointers to private data that (i) point to a single known location where the private data is stored and (ii) point to a memory pool or a number of locations where private data is stored and the location of the private data is not known. In determining how this can be implemented in a C-like programming language, we considered pre-allocating memory pools for pointers with private locations. Such memory pools would be required for each data type to ensure that we can store and extract private data correctly. This approach, however, has severe disadvantages, which are:

(1) Using memory pools unnecessarily increases the program's memory footprint, where one pool will be needed for each used data type including complex types defined via the struct construct. Furthermore, it is not clear to what size each pool should be set to optimize performance.

(2) It would also often incur unnecessarily large computation costs due to the need to touch all locations within a memory pool per single access (or touch several locations when the pool is implemented using more complex ORAM techniques). As will be evident later in the article, there are large classes of programs, applications, and data structures, where a pointer to private data always corresponds to a single location, which removes the need to use secure multi-party computation techniques for pointer manipulation. Allowing a pointer to store a single known location drastically improves program performance compared to using pointer pools.

(3) Memory pools would also not work in the presence of pointer casting.

Then, if we do not want a pointer to initially point to a pre-allocated memory pool, would the decision to properly declare a pointer as pointing to a single (known) location or a set of locations 
be left to the programmer? This is going to introduce an additional burden for a programmer who would need to know at a variable declaration time whether the variable of a pointer type will require protecting its value. This happens if the pointer is used inside a conditional statement with private condition, which then requires protecting the location assigned to the pointer to protect the result of the condition evaluation.

To ease the programming burden and at the same time avoid consuming unnecessary (memory and computation) resources, our solution is to use the same programming interface for all pointers that are to point to private data. When the pointer is being declared or initialized, it has one known location associated with it (if the pointer is not initialized, that location is set to the default value corresponding to uninitialized pointers). Throughout the computation, the pointer, however, may be pointing to multiple locations, one of which is its true location. This happens when the pointer's value is modified inside conditional statements with private conditions as illustrated next. Suppose we declare variables $a$ and $b$ to be private integers followed by the code below:

1. private int $* \mathrm{p}$;

2. $\mathrm{p}=$ \&a;

3. if (priv-cond) then $p=\& b$;

We see that variable $p$ was declared as a pointer to a private integer, but the type of the pointer with respect to whether the location itself is private is implicit. After executing lines 1 and $2, p$ has a single known location, but after executing line $3, \mathrm{p}$ is associated with a list of two locations (the address of $a$ and the address of $b$ ) and the value of the true location is protected. That is, a pointer always starts with a single publicly known location and the location to which it is pointing may become private, but the user does not declare the pointer itself as public or private. In the rest of this work, we use the term "public location" in reference to a pointer to private data to mean that the pointer has a single known location (either initialized or uninitialized), and we use the term "private location" to mean that the pointer has a list of public locations, but which location is in use remains private.

When we consider interaction of public and private values in connection to the use of pointers, a number of questions arise, which we address next.

(1) Can a pointer that was declared to point at private data be assigned the address of public data? Note that without the use of pointers, the equivalent actions are generally allowed. That is, a variable declared to hold private data can be assigned a known value, which is consequently converted into protected form. The same does not hold for pointers, and we disallow assigning locations of public variables to pointers that were declared to point to private data. To see why, suppose that a user program contains the code below where a was declared to be a public integer, while $b$ is a private integer:

1. $p=\& a$;

2. if (priv-cond) then $p=\& b$;

3. $* p+=1$;

After executing lines 1 and 2, p stores two addresses and the true location of where it is pointing out of these two addresses is protected. On line 3 , however, the pointer is dereferenced and the result of private condition priv-cond evaluation is revealed by examining the value of a before and after line 3 . Thus, to eliminate information leakage, pointers to private data can be assigned only locations that store private values.

(2) Can a pointer declared to point to public data be modified inside conditional statements with private conditions and as a result become pointing to multiple locations? The answer to this question is No. If a pointer to public data is updated in the body of a conditional statement with private condition, then it must be treated as a pointer to private data (otherwise, 
using its dereferenced value reveals unauthorized information). Allowing such uses and performing the conversion implicitly by the compiler will be confusing to the programmer (who no longer can use the pointer to store addresses of public data). For that reason, we disallow updates to pointers to public data within the body of conditional statements with private conditions.

We note that all program transformations that we describe in this work preserve semantics of the original program and, given that a program can be compiled into the corresponding secure implementation, the transformed program will always produce the same output as the original program. There are some restrictions that user programs must meet to be compiled into secure implementations with no information leakage. Such restrictions include the two cases at the interaction of public and private data described above and additional mixed-mode restrictions placed on programs with or without pointers (e.g., the fact that the body of a conditional statement with a private condition cannot have public side effects, a loop termination condition should be public or made public, etc.). This is to ensure that no information leakage in the compiled program can take place, and the programs that do not meet the requirements are aborted at compilation time. Once these security constraints are met, our extension of PICCO with pointers will allow any user program to be compiled into its secure counterpart.

We next proceed with describing how pointers to private data are implemented to realize the ideas outlined above. We start with general description applicable to different underlying secure multi-party computation frameworks.

\subsection{Pointer Representation}

As we incorporate support for pointers, we first note that pointers to public data will not need to be modified and their implementation remains the same as in $\mathrm{C}$. The most significant change in implementing pointers to private data comes from the need to maintain multiple locations. For that reason, the data structure that we maintain for pointers to private data consists of (i) an integer field that stores the number $\alpha(\geq 1)$ of locations associated with the pointer; (ii) a list $L$ of $\alpha$ addresses where the data is stored; and (iii) a list $T$ of $\alpha$ private tags, one of which is set to 1 (true data location) and all others are set to 0 . For the important special case of $\alpha=1$, the pointer has known (public) location and the tags are not used.

We formalize the above pointer representation using the following invariant, which is maintained throughout various pointer operations: among all locations stored with a pointer to a private object, there is exactly one true location of the object and the tag corresponding to that location is set to 1 , while the tags corresponding to all other locations are set to 0 . This invariant is true of all well-formed programs and may be violated only in the case of dangling pointers as detailed later.

Because we will employ a uniform data structure for pointers to private data of any data type such as integer, floating-point values, and so on, and even pointers to a pointer, the data structure we maintain needs to include two additional fields: (iv) an integer flag that determines the type of data associated with the pointer (i.e., integer $=1$, float $=2$, struct $=3$, etc.) and (v) an integer field that indicates the indirection level of the pointer. For instance, if a pointer refers to a private value of a non-pointer type, its indirection level is set to 1 ; and if it refers to a pointer whose indirection level is $k$ (for $k \geq 1$ ), its level will be set to $k+1$. A pointer to a struct also has indirection level 1 regardless of the types of the struct's fields (which can be pointers themselves).

\subsection{Pointer Updates}

Initially, at a pointer declaration time, the number of locations $\alpha$ associated with the pointer is set to 1 and the address is set to a special constant used for uninitialized pointers. Then, every time 
the pointer is modified (including simultaneously with pointer declaration), its data structure is updated. When the pointer is assigned a new location using a public constant, a variable's address, or a memory allocation mechanism (e.g., as in $p=0, p=\& a$, or $p=\operatorname{malloc}(\operatorname{size})$ ), $\alpha$ in the pointer's data structure is set to 1 and the associated address is stored in the pointer's address list. When a pointer is updated using another pointer ( $a$ in $p=p 1$ ), the latter's data structure is copied and stored with the former.

Such simple manipulations are used only when the assignment does not take place inside the body of a conditional statement with a private condition. Pointer assignments inside conditional statements with a private condition present the most interesting case when the list of pointer locations gets modified. Updating values modified in the body of a conditional statement with a private condition already requires special handling in PICCO and other compilers, and all we need is to support a specific procedure when a variable of a pointer type is being modified. We need to distinguish between if-then and if-then-else statements, which we consequently discuss.

Consider the following code with an if-then statement:

1. $\mathrm{p}=\mathrm{p} 1$;

2. if (priv-cond) then $p=p 2$;

where $p, p 1$, and $p 2$ are pointers (to private data) of the same type. This is the most general case, where on line 2 both $\mathrm{p}$ and $\mathrm{p} 2$ can have any number of locations associated with each of them (recall that all other assignment types use a single location). When this code is written for ordinary (private) variables of the same type $a, a_{1}$, and $a_{2}$, a generic way to implement this update is to first set $[a]=\left[a_{1}\right]$ and then compute $[a]=\left([c] \wedge\left[a_{2}\right]\right) \vee(\neg[c] \wedge[a])$, where $c$ is a bit equal to the result of evaluating priv-cond and notation $[x]$ means that the value of $x$ is protected and its value is not known throughout the computation. (Note that this operation can be optimized as $[c]$. $\left(\left[a_{2}\right]-[a]\right)+[a]$ using secret sharing or $\left([c] \wedge\left(\left[a_{2}\right] \oplus[a]\right)\right) \oplus[a]$ using garbled circuits. $)$ In the case of pointers, such a simple update does not work, because this procedure would turn addresses into secret shared values preventing the pointer from being dereferenced (without touching all possible memory locations). Thus, after executing the assignment $p=p 1$, we combine the (public) locations of $p$ and $p 2$ and set the tags in $p$ based on the current tags of $p$ and $p 2$ and the result $c$ of evaluating priv-cond. Let pointer $\mathrm{p}$ after executing the first assignment contain $\alpha_{1}$ locations stored as $L_{1}=\left\{\ell_{1}, \ldots, \ell_{\alpha_{1}}\right\}$ with corresponding tags $T_{1}=\left\{\left[t_{1}\right], \ldots,\left[t_{\alpha_{1}}\right]\right\}$ (i.e., this information was copied from $\mathrm{p} 1)$. Let also $\mathrm{p} 2$ store $\alpha_{2}, L_{2}=\left\{\ell_{1}^{\prime}, \ldots, \ell_{\alpha_{2}}^{\prime}\right\}$, and $T_{2}=\left\{\left[t_{1}^{\prime}\right], \ldots,\left[t_{\alpha_{2}}^{\prime}\right]\right\}$. Note that the addresses in each $L$ can be listed in any order (as long as the ordering is consistent across all computational parties), but the tag $t_{i}$ in $T$ must correspond to the address $\ell_{i}$ at the same position $i$ in $L$. Then, as a result of the conditional assignment, we compute p's new content as given in Algorithm 1.

In the algorithm, $L_{3}$ is composed of all locations appearing in $L_{1}$ or $L_{2}$ (repeated locations are stored only once). We use notation $L$.find to retrieve the position of the element of $L$ provided as the argument or special symbol $\perp$ if the element is not found. The tags in the output $T_{3}$ are set based on three different cases: (i) a location in $L_{3}$ is found in both $L_{1}$ and $L_{2}$; (ii) it is found in $L_{1}$, but not in $L_{2}$; and (iii) it is found in $L_{2}$, but not $L_{1}$. Because only tags in $T_{1}$ and $T_{2}$ and $c$ are private, only lines 7, 9, and 11 correspond to private computation.

If the conditional statement is of the form if-then-else, but $\mathrm{p}$ is not updated in the body of the else clause, then the computation in Algorithm 1 is applied unchanged. If the pointer is instead updated only in the body of the else clause, then the computation is performed similarly, but Algorithm 1 is called with the value of $1-c$ instead of $c$.

Last, if the pointer is updated in both clauses of the if-then-else statement, the pointer content prior to that statement needs to be disregarded. The pointer values used in the two assignments 


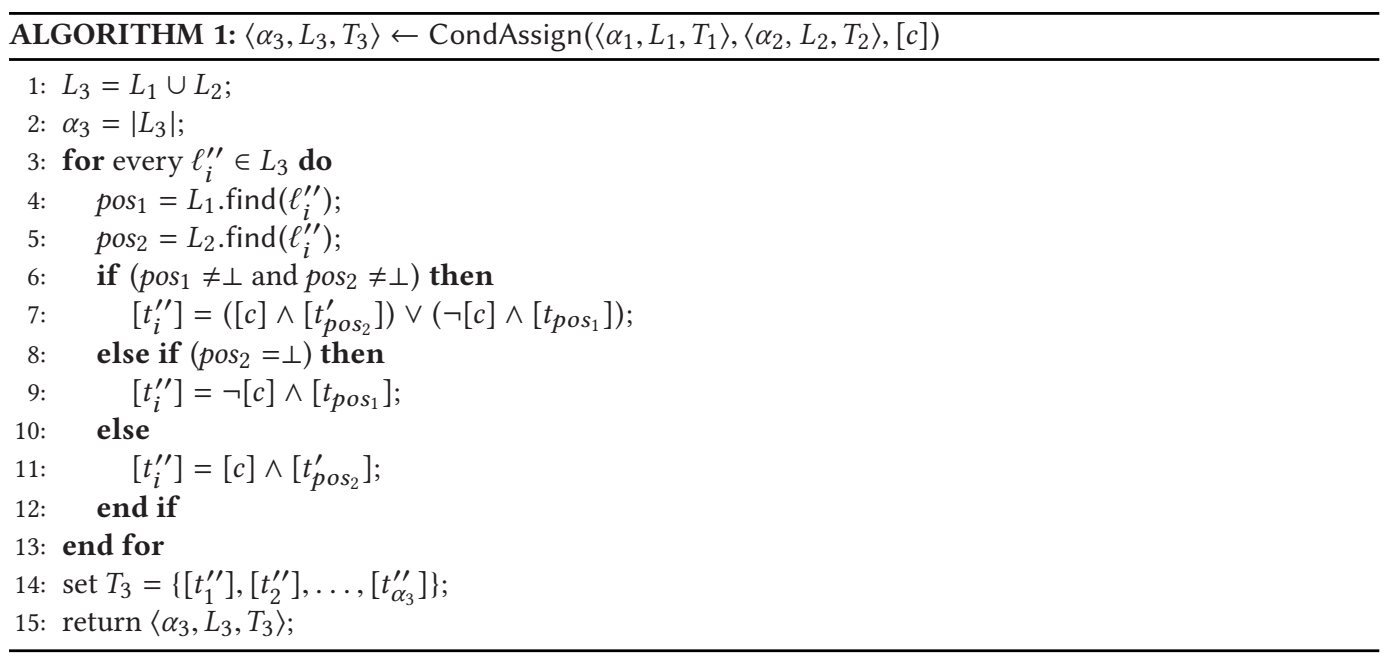

are then merged as in Algorithm 1 using the result $c$ of the private condition evaluation. To better illustrate this, consider the following code segment:

1. $\mathrm{p}=\mathrm{p} 1$;

2. if (priv-cond) then $p=p 2$;

3. else $p=p 3$;

After we assign $p 1$ to $p$ on the first line, p's content is overwritten with the content of either p2 or p3 depending on the result $c$ of evaluating priv-cond. We can see that before entering the if-clause, the current content of $p$ (i.e., that copied from $p 1$ ) can be safely disregarded without affecting its correctness. In other words, to update $\mathrm{p}$ inside the conditional statement, we call CondAssign $\left(\left\langle\alpha_{2}, L_{2}, T_{2}\right\rangle,\left\langle\alpha_{3}, L_{3}, T_{3}\right\rangle, c\right)$ in Algorithm 1, where $\left\langle\alpha_{2}, L_{2}, T_{2}\right\rangle$ and $\left\langle\alpha_{3}, L_{3}, T_{3}\right\rangle$ are contents of pointers $\mathrm{p} 2$ and $\mathrm{p} 3$, respectively.

These constructions compose in the presence of nested conditional statements with private conditions. For instance, after executing the code:

1. if (priv-cond1) then $p=p 1$;

2. else

3. $\mathrm{p}=\mathrm{p} 2$;

4. if (priv-cond2) then $p=p 3$;

5. else $\mathrm{p}=\mathrm{p} 4$;

$p$ will contain the combined content of pointers p1, p3, and p4. That is, Algorithm 1 is first called with the content of pointers $\mathrm{p} 3$ and $\mathrm{p} 4$ and the result $c_{2}$ of evaluating priv-cond2, after which Algorithm 1 is called on the result of its previous execution, the content of $\mathrm{p} 1$, and the result $c_{1}$ of evaluating priv-cond1.

\subsection{Pointer Dereferencing}

When pointer $\mathrm{p}$ with a private location is being dereferenced, its dereferenced value is privately computed from $\alpha, L=\left\{\ell_{1}, \ldots, \ell_{\alpha}\right\}$, and $T=\left\{\left[t_{1}\right], \ldots,\left[t_{\alpha}\right]\right\}$ stored at $p$ in a logical way. Let $\left[a_{i}\right]$ denote the value stored at location $\ell_{i} \in L$ for pointer with indirection level of any type (simple type, struct, function, etc.). Then, we compute the dereferenced value as $[v]=\bigvee_{i=1}^{\alpha}\left[a_{i}\right] \wedge\left[t_{i}\right]$.

When the dereferenced value is being updated, all locations in $L$ need to be touched, but the content of only one of them is being changed. If we, as before, use $\left[a_{i}\right]$ to denote the value stored 
at $\ell_{i} \in L$ and let $\left[a_{n e w}\right]$ denote the value with which the dereferenced value is being updated, then we update the content of each location $\ell_{i}$ as $\left[a_{i}\right]=\left(\left[t_{i}\right] \wedge\left(\left[a_{n e w}\right]\right) \vee\left(\neg\left[t_{i}\right] \wedge\left[a_{i}\right]\right)\right.$. That is, the true location $\left(t_{i}=1\right)$ will be set to $a_{n e w}$, while all others $\left(t_{i}=0\right)$ will be kept unchanged.

Because a pointer to private data is represented using a special structure, we need to modify the procedures above for pointers with indirection level $>1$ (i.e., pointers to pointers) to correctly assemble and update pointer objects. In this case, each $\ell_{i} \in L$ stores an address of a pointer $p_{i}$ and let each $p_{i}$ be associated with $\alpha_{i}, L_{i}=\left\{\ell_{1}^{(i)}, \ldots, \ell_{\alpha_{i}}^{(i)}\right\}$, and $T_{i}=\left\{\left[t_{1}^{(i)}\right], \ldots,\left[t_{\alpha_{i}}^{(i)}\right]\right\}$. To retrieve the dereferenced value of $\mathrm{p}$, we need to compute $\left[t_{i}\right] \wedge\left[t_{j}^{(i)}\right]$ for $1 \leq i \leq \alpha$ and $1 \leq j \leq \alpha_{i}$ and merge all lists $L_{i}$ for $1 \leq i \leq \alpha$. The resulting list is thus set to $L^{\prime}=L_{1} \cup L_{2} \cup \cdots \cup L_{\alpha}$ and let $\alpha^{\prime}=\left|L^{\prime}\right|$. For any location in $L^{\prime}$, its corresponding tag is the OR of all $\left[t_{i}\right] \wedge\left[t_{j}^{(i)}\right]$ values matching that location in the individual lists $L_{i}$. The result is $\alpha^{\prime}, L^{\prime}$ and the corresponding tags $T^{\prime}$.

To illustrate this on an example, let $\alpha=3, T=([0],[0]$, [1]), and $L$ store the addresses of pointers $p_{1}, p_{2}, p_{3}$ with $\alpha_{1}=1, L_{1}=(123), T_{1}=(1) ; \alpha_{2}=2, L_{2}=(189,245), T_{2}=([0],[1]) ;$ and $\alpha_{3}=3, L_{3}=$ $(123,176,207), T_{3}=([0],[1],[0])$. The result of this operation is a pointer with $L^{\prime}=L_{1} \cup L_{2} \cup L_{3}=$ $(123,176,189,207,245), \alpha^{\prime}=\left|L^{\prime}\right|=5$, and $T^{\prime}=([0],[1],[0],[0],[0])$.

To update the dereferenced value of $\mathrm{p}$ through an assignment as in $\star \mathrm{p}=\mathrm{p}$ ', each pointer $p_{i}$ stored at address $\ell_{i} \in L$ needs to be updated with p' 's information. In particular, for each $p_{i}$ each $\operatorname{tag}\left[t_{j}^{(i)}\right]$ (for location $\ell_{j}^{(i)}$ ) is updated to $\left(\neg\left[t_{i}\right]\right) \wedge\left[t_{j}^{(i)}\right]$. We also compute tag $\left[t_{i}\right] \wedge\left[t_{j}^{\prime}\right]$ for each location $\ell_{j}^{\prime}$ in $\mathrm{p}$ 's list of locations. We then merge the location list of each $p_{i}$ with that of $\mathrm{p}$ ' to form $p_{i}$ 's new list. For any new location inserted into $L_{i}$, its tag is set to the computed $\left[t_{i}\right] \wedge\left[t_{j}^{\prime}\right]$ for the appropriate choice of $j$, and any location that appears on both $p_{i}$ and p' lists, the value $\left[t_{i}\right] \wedge\left[t_{j}^{\prime}\right]$ is ORed/added to $p_{i}$ 's updated tag for that location. In other words, if $t_{i}$ is true, we take $p$ 's value and otherwise keep $p_{i}$ 's value.

If pointer $\mathrm{p}$ with a private location is being dereferenced $m>1$ times, then the above dereference algorithms are naturally applied multiple times with the first $m-1$ instances being the version that produces a pointer and the last instance producing either a pointer or a private value depending on p's indirection level. $p$ can then be treated as the root of a tree with its child nodes being locations of pointers stored in its list and the leaves of the tree eventually pointing to private data (of a nonpointer type). To perform an $m$-level dereferencing operation, we traverse the top $m+1$ levels of the tree and consolidate the values stored at those levels (and update the values at the $(m+1)$ th level if the dereferenced value is to be updated).

\subsection{Pointers to Struct}

We next discuss design and implementation of pointers to structs, including their representation and the associated algorithms. Pointers to complex data types declared using struct constructs are common for building data structures such as linked lists, stacks, and trees, and thus pointers to structs deserve special attention.

As before, if a complex data type contains no private fields, no transformations are needed. However, when dealing with pointers to structs with private fields, we need to address the following questions:

(1) A struct groups together a number of different variables that can be either private or public, but the complex data type itself declared using struct is not associated with any particular type of secrecy. When declaring a pointer to a complex data type, we thus need to determine if a pointer to it can be treated as a pointer to private data or if it has to be treated as a conventional pointer to a public variable.

(2) When designing the representation of a private pointer that points to struct, we need to take into account the fact that fields of a complex data type can be accessed and modified 
independently of each other or the struct itself. Thus, it remains as a question whether we should maintain a separate list of addresses for each struct field or maintain only a single list of addresses for all possible struct variables associated with the pointer.

(3) The last question is whether we can reuse the previously described algorithms for working with private pointers for updating or dereferencing pointers to structs on the individual fields of a struct or if modifications are needed.

In what follows, we thus focus on answering these questions.

Secrecy of pointers to struct. Secrecy of a pointer to struct is implicitly determined by the protection modes of the struct's fields. We determined that a pointer to a complex data type can be treated as a pointer to private data only if all fields in its declaration are private. It means that if at least a single field of a struct is public, pointers to this data type can be of public type only. This treatment is necessary to eliminate information leakage when pointers to structs are modified inside conditional statements with private conditions. Consider, for example, a data type containing one private and one public field. If we treat a pointer to this data type as a pointer to private data, then it can be modified inside an if-statement with a private condition and have multiple locations associated with the pointer. However, by dereferencing and observing the value of the public field, one can determine the true location of the pointer and thus learn unauthorized information about the result of the private condition.

Because a complex data type may contain other struct variables as its fields, the variables in the data type will need to be checked recursively to determine whether at least one public field is present (with provisions to skip cycles in the declarations). If none are found, then pointers to this data type are treated as pointers to private data.

Pointer to struct representation. To implement private pointers to structs, we needed to determine whether a single list of locations is sufficient for all fields of the complex data type (recall that all fields are private) or separate lists must be maintained. In working to answer this question, we determine that there is no need to maintain multiple lists of locations, because the list of locations associated with each field in the struct must be the same (adjusted for the offset of the field within the struct). That is, values of a struct's fields can be modified individually (e.g., as in $p->x=y$ ), but the only way to access or modify the location of a field is through the location of the entire struct. In other words, the list of addresses associated with a pointer to struct $p$ (and thus the addresses corresponding to all of its fields) can be modified only by directly updating $p$, as operations of the type $p->x=y$ do not affect the list of addresses associated with the field $x$. Storing a single list has the added benefit that we can employ the same representation of pointers to private data as for simple data types. This treatment also implies that a pointer to a struct object will have indirection level 1 even if all fields of the struct are pointers themselves.

Operations on private pointers to struct. We represent pointers to a struct record in the same way as other pointers. This means that operations for using pointers and updating their values remain unchanged. To dereference a specific field of a pointer as in $p->x$ and retrieve the value of the variable $x$, also only minor changes to the previously described algorithms are needed. In particular, all we need is to determine the offset $f$ of the variable's address within the record and perform the dereferencing procedure in the same way as for pointer $p$ itself, but instead of using locations $\ell_{i}$ from $L$, we use locations $\ell_{i}+f$. The same modification applies to the case when the dereferenced value is modified through assignment.

If we would like to dereference $p$ and retrieve the entire record as in $r e c=\star p$, then we need to iterate through each field of the struct and retrieve the dereference value of each field as described above for $p->x$. Similarly, to update a dereferenced pointer $p$ as in $\star p=r e c$, we need to perform the equivalent of $p->x=r e c . x$ for each field $x$ of the struct. 


\subsection{Pointers to Functions}

Similar to pointers to ordinary data types, we need to distinguish between pointers to functions that will be treated as pointers to private data and pointers to functions that will be treated as pointers to public data. The former can be used inside conditional statements with private conditions (as a result of which they acquire multiple locations and the true location becomes private) and are restricted to functions with no public side effects. The latter can contain pointers to functions of any type, but cannot be modified or dereferenced inside conditional statements with private conditions. The distinction is made at the time of pointer declaration using private/public qualifiers with void data type. That is, by using private void $* p, p$ will be treated similar to other pointers to private data, while all pointers declared syntax public void $\star p$ will be treated as conventional C pointers.

Private pointers to functions are supported naturally in our framework. When a pointer stores a single location, the function is invoked as in conventional program execution. If, however, a pointer acquires multiple locations as a result of its modification inside conditional statements with private conditions, then at the time of pointer dereferencing all functions stored in the pointer will be executed, but only the effects of one of them will be applied. Conceptually, this is the same as executing branching statements with private conditions: all branches are executed, but only the effects of one of them are applied depending on the result of private condition evaluation. That is, when a pointer $\mathrm{p}$ storing $\alpha$ locations $L=\left(\ell_{1}, \ldots, \ell_{\alpha}\right)$ with the corresponding tags $T=\left(t_{1}, \ldots, t_{\alpha}\right)$ is being dereferenced, each function $f_{i}$ stored at address $\ell_{i}$ is being invoked. Then, each (private) variable $a$ that $f_{i}$ modifies is set to $a=\left(a_{i} \wedge t_{i}\right) \vee\left(a_{\text {orig }} \wedge \neg t_{i}\right)$, where $a_{\text {orig }}$ and $a_{i}$ are its original and newly computed by $f_{i}$ values. If $a$ is modified by multiple $f_{i}$ 's with indices $i_{1}$ through $i_{k}$, then its value is updated as $a=\left(\bigvee_{j=1}^{k} a_{i_{j}} \wedge t_{i_{j}}\right) \vee\left(a_{\text {orig }} \wedge \neg \bigvee_{j=1}^{k} t_{i_{j}}\right)$ (recall that only one $t_{i}$ can be set to 1 , so we can replace the ORs in the formula with addition or XOR for efficiency).

\subsection{Pointer Predicates}

As previously discussed, the value of a pointer to private data is treated as public when it stores a single location $(\alpha=1)$, and it is private otherwise $(\alpha>1)$. More generally, if pointers to private data are used in predicates or similar expressions, the result of a predicate evaluation is public if its outcome can be determined using only public data. For example, the outcome of an expression that compares two pointers to private data for equality is public if (i) both pointers store a single location in their lists $L_{1}$ and $L_{2}$ or (ii) at least one of the pointers stores multiple locations, but $L_{1} \cap L_{2}=\emptyset$. In other circumstances, the outcome depends on private tags and is treated as private. Note that when the result of a predicate evaluation on pointers is private, it can be naturally computed by privately determining the true location of each pointer and applying the predicate to them.

Because it is not always possible to determine at compile time whether a predicate evaluated on one or more pointers (to private data) will have a public or private status (which, for example, may depend on program's public input), some checking will need to be deferred to run time. We show how we address this in PICCO in Section 6.1.

\subsection{Passing Arguments by Reference}

Function calls contribute to the basic software engineering principles of modular program design, but could be expensive in terms of stack memory usage for the passed arguments. This has led to differentiating between function calls where the arguments are passed by value and by reference. In the latter case, the function typically takes a pointer to the argument and all updates to the 
dereferenced pointer will be visible after completing the function call (thus, arguments passed by reference can be used for either input or output).

Passing private variables to functions by reference inherits the same benefits as for conventional (public) variables in the programming language. The good news is that no special provisions are needed for passing private variables by reference, resulting in efficient implementations. Furthermore, because often to pass an argument by reference, its address is supplied to a function call (as opposed to supplying an existing pointer), the resulting pointer will have a single known location. This allows us to enjoy the benefits of avoiding using extra resources without the slowdown of working with pointers with private locations.

\subsection{Array Indexing}

Because arrays are based on pointers, array indexing also applies to pointers. Thus, we can see constructions such as $p=a$ and $p[i]$, where $p$ is a pointer and $a$ is an array, and need to support them for pointers to private data. Pointer indexing $\mathrm{p}[\mathrm{i}]$ with a pointer $\mathrm{p}$ to private data and a public index $i$ is implemented naturally, where we iterate through all locations in the address list $L$ of $p$, advance each of them by $i$ multiplied by the size of the data type, retrieve the data at the determined positions, and combine all of them using private tags for each location to obtain the result. In other words, the computation is very similar to that of pointer dereferencing, where instead of retrieving data at the positions specified in $L$, we advance each position by $i$ data items. (As $C$ permits the use of negative indices, when $i$ in $p[i]$ is negative each location in $L$ is decremented by the necessary amount during this operation.)

\subsection{Pointer Arithmetic}

Pointers can be modified by setting the address to which they point to the result of an arithmetic expression evaluation. While in $\mathrm{C}$ pointers can be used in arbitrary expressions similar to the way integer variables are used, only a limited set of operations on pointer variables is meaningful when they are used to store and manipulate addresses within the program. For example, pointer arithmetic can be relied upon to increment or decrement a pointer value by an integer amount to move to a different position within an array or between struct fields, which can also be achieved without using pointer arithmetic. Many other arithmetic operations on pointer variables are not meaningful, and moving between different variables using pointer arithmetic is unreliable and error-prone. Nevertheless, not supporting pointer arithmetic makes the approach deviate from standard $\mathrm{C}$ and disables a small class of functionalities such as, for example, embedded linked lists as implemented in the Linux kernel. Thus, we identify meaningful operations on pointers to private data and realize them in this framework as described next.

As mentioned before, in regular $\mathrm{C}$, pointers can store any integer values and using pointer variables in arithmetic expressions will result in evaluating the expressions on the integer values stored in such variables. In the context of pointers to private data, we, however, distinguish between pointer objects and (integer) addresses that pointer objects store. Thus, pointer content is no longer equivalent to integer values, and we support pointer arithmetic with pointer objects only for the purposes of using pointers to store and manipulate addresses. Such arithmetic operations can be categorized into two groups:

(1) Using pointers to private data in expressions of the type $p+\exp$ and $p-\exp$, where exp is an expression that evaluates to a (public) integer value. This operation produces the same output as executing \&p[exp], i.e., all locations stored in $p$ are advanced by the amount of space occupied by exp elements of the array. 
(2) Using pointers to private data in offset computation as in $\mathrm{p} 1$ - p2. This operation is straightforward to implement when both pointers store a single location. When, however, at least one of them has multiple locations, we realize this operation by computing the private difference between the true locations of the pointers. This option, in our opinion, implements the right semantic value as opposed to other variants (such as computing pairwise differences between all addresses stored in the pointers) that bear little meaning.

Note that expressions of the type $p+\exp$, and equivalently \&p[exp], where exp evaluates to a private integer, are not meaningful and not supported.

\subsection{Pointer Casting}

Variable casting refers to the ability to treat a variable of one type as a variable of another type. Casting a constant or variable of one type to a constant or variable of another type typically results in the value being preserved after the conversion (if possible) even if the two types use different data representations. This means that conversion is likely to involve computation. Conversion algorithms between floating point and integer values in this framework are known (see, e.g., Reference [2]), while conversion between integer types of different sizes and floating point types of different sizes requires minimal to no work (assuming no overflow or underflow detection is required when casting a value to a shorter representation).

Pointer casting is handled differently and $\mathrm{C}$ is unique in the sense of allowing pointer-based inmemory casting from one data type to another. Pointer casting involves no data conversion: the memory is read as is and is interpreted as a sequence of elements of another type. Thus, pointer casting is meaningful between a limited number of data types. To support pointer casting, we need to resolve the main question: because data representation of private data types differs from data representation of the corresponding public data types, we need to determine how to mimic sizes of public data types when working with blocks of private data without modifying the data itself. For example, a secret sharing implementation represents private values as elements of the same field, which means that, for example, shares of a 16-bit integer and shares of a 64-bit integer have the same bitlength. However, a programmer who casts memory storing an array of 64-bit integers to a pointer to an array of 16-bit integers, expects to extract four 16-bit integers from each 64-bit integer. This means that to meet the programmer's expectations, private data will need to be processed and assembled in a different form. We, however, cannot modify the original data, because only the pointer was cast, not the data itself.

Instead of duplicating the memory and performing conversion at the time of casting, our solution is to do the necessary computation at the time of pointer dereferencing. This means that we need to record information about the data type from which casting was performed (to the data type of the pointer) at the time of casting, but delay conversion until the pointer is dereferenced. We store casting data type information with the pointer and use it to extract the relevant portion of the memory at pointer dereferencing time. Note that in the presence of a sequence of casts, only a single data type needs to be maintained, because the memory layout does not change.

\section{DYNAMIC MEMORY MANAGEMENT}

Pointers are often used in programming to dynamically allocate memory on the heap and deallocate it when it is no longer in use. Here, we focus on C-style malloc() and free() used with pointers to public variables and show what modifications are needed to support dynamic memory allocation with pointers to private variables.

malloc() in $\mathrm{C}$ allocates the requested number of bytes on the heap that are passed as an argument to the function malloc(). The result of this function is the address of the allocated variable 
or the first array element in case of dynamic array allocation, which is stored in a pointer. To support dynamic memory allocation for private variables, we start with the following code in C:

1. int* $\mathrm{p}=($ int $*)$ malloc $(\operatorname{sizeof}($ int $))$;

2. int* $\mathrm{p} 1=($ int $*)$ malloc $(10 * \operatorname{sizeof}($ int $))$;

Here, $\mathrm{p}$ points to single variable, while $\mathrm{p} 1$ points to a dynamic array of size 10 . The assignment operator directly saves the malloc result into the pointer, because they are of compatible types. However, this is not the case for pointers to private variables, because a private pointer is represented using multiple fields. Consequently, we cannot assign the malloc result directly to a private pointer and use a modified interface for pointers to private variables. In particular, we use a function pmalloc ${ }^{2}$ to implement private malloc, which is invoked as

1. private int* $\mathrm{p}=\operatorname{pmalloc}(10$, private int);

As shown, pmalloc takes two arguments, which are the requested number of dynamic variables and the data type. The function returns the data structure used for private pointers in our implementation with $\alpha=1$ and the only location in $L$ set to the address of the first variable in the allocated array (when the first argument to the function is $>1$ ). Specifying the private data type is often necessary to properly allocate and initialize the memory. For example, in PICCO a private integer is represented using one variable of type mpz_t from the GMP library [1] and a private float is represented using four $\mathrm{mpz} z_{-} t$ variables. Once memory for the necessary number of variables is allocated, each of them also needs to be initialized before it can be used in computation.

Calling free with a pointer in $\mathrm{C}$ allows to deallocate the memory (for either a variable or dynamic array) to which the pointer is pointing. To support similar functionality for private variables, we design a function pfree that similarly takes a pointer (to a private variable or dynamic array) as its only argument. With pfree, we distinguish between two different cases: the pointer provided as an argument to the function has a single known location (i.e., $\alpha=1$ ) or it has a private location out of a public list $(\alpha>1)$.

Handling the first case is simple and efficient: we can simply call free to deallocate memory associated with the address stored in the pointer. Pointers to private data with public locations constitute a very common use case in programs that use pointers to private data or build data structures from private data (e.g., linked lists, stacks). Freeing memory used by pointers to private data in such cases is thus going to be extremely efficient and does not introduce additional overhead.

Handling the second case well, however, is very challenging. This is because deallocating physical memory results in publicly observable outcomes, and we must be extremely careful not to reveal the true location stored in a pointer with a private location while at the same time reducing the program's memory usage. For example, a simple strategy of deallocating memory associated with all locations on a pointer's list of addresses will not be acceptable for some programs. That is, consider a dummy example with two pointers $\mathrm{p} 1$ and $\mathrm{p} 2$, for each of which we allocate memory using pmalloc. Then, the locations to which the pointers are pointing are swapped based on the result of a private condition evaluation. We obtain that both $\mathrm{p} 1$ and $\mathrm{p} 2$ now contain two identical locations in their lists of addresses, but their true addresses are distinct. Suppose we process the data to which $\mathrm{p} 1$ points and want to deallocate the corresponding memory. If we deallocate both addresses on $\mathrm{p} 1$ 's list, then $\mathrm{p} 2$ becomes a dangling pointer and the data to which it was pointing is no longer accessible. Thus, such an implementation of pfree would be too restrictive to permit its general use.

\footnotetext{
${ }^{2}$ Note that the choice of the function is not crucial and it can be called malloc instead to simplify programmers' effort for transforming an existing program to an equivalent program that computes with private data. We, however, prefer to use pmalloc to make it explicit that the computation refers to private data.
} 
Thus, calling pfree $(p)$ should result in deallocating memory associated with only one address on p's list of addresses. Furthermore, the address being deallocated cannot depend on any private data (but can be any function of public data). This means that we are not necessarily deallocating memory associated with the true location of the pointer and other pointers that store the same location on their lists must be adjusted to preserve correctness of the computation (which involves additional resources). For example, we can choose to deallocate the fist location $\ell_{1}$ on a pointer's list, but if this was not the pointer's true location (which we can privately check), the data stored at $\ell_{1}$ needs to be relocated and other pointers storing $\ell_{1}$ on their lists need to be updated accordingly. We next describe in more detail how we can realize this idea.

First, if the pointer $\mathrm{p}$ on which pfree was called contains the default location corresponding to uninitialized pointers on its list of addresses $L$ (which is public knowledge), we choose not to perform memory deallocation. This is to ensure that no memory is being deactivated (which may be in use by other pointers) if $\mathrm{p}$ happens to be uninitialized. Otherwise, we free the first location $\ell_{1}$ on p's list. ${ }^{3}$ (Alternatively, the location used by the smallest number of pointers can be freed.) Before we can actually free the memory, we need to privately update the values stored at the remaining locations in $L$ using the value stored at $\ell_{1}$ to maintain correctness. We will need to ensure that (i) if $\ell_{1}$ happens to be the true location, the values stored in the remaining locations will remain unchanged and (ii) if $\ell_{1}$ is not the true location, the value stored at $\ell_{1}$ can be found at p's true location, while the values stored at all other locations remain unchanged. The rationale for doing this as follows: if $\ell_{1}$ is indeed p's true location, no additional work would be required if this fact was public (i.e., it is the programmer's job to ensure that freeing $p$ does not affect other variables still in use). If $\ell_{1}$, however, was not p's true location, then it may be in use by other pointers and the value stored at $\ell_{1}$ needs to be relocated to p's true location prior to memory deallocation (and the pointers that contain $\ell_{1}$ in their lists need to be updated accordingly).

Let $\mathrm{p}$ at the time of calling pfree store $\alpha, L=\left\{\ell_{1}, \ldots, \ell_{\alpha}\right\}, T=\left\{\left[t_{1}\right], \ldots,\left[t_{\alpha}\right]\right\}$ and $A=$ $\left\{\left[a_{1}\right], \ldots,\left[a_{\alpha}\right]\right\}$ denote values stored at locations in $L{ }^{4}$ To obliviously update $\left[a_{i}\right]$ 's for $2 \leq i \leq$ $\alpha$, we compute $\left[a_{i}\right]=\left(\left[t_{i}\right] \wedge\left[a_{1}\right]\right) \vee\left(\neg\left[t_{i}\right] \wedge\left[a_{i}\right]\right)$. This satisfies the above two requirements as follows: if $t_{1}$ is true ( $\ell_{1}$ is the true location) and thus $t_{i}$ is false, the result will be $a_{i}$ for any $i$; if $t_{i}$ is true and thus $t_{1}$ is false, the result will be $a_{1}$; if both $t_{1}$ and $t_{i}$ are false, the result will be $a_{i}$. Surprisingly, the formula does not depend on $t_{1}$.

Second, we need to update private pointers that store the freed location $\ell_{1}$ in their lists (and are still in use), but no computation needs to be performed for pointers that store any of $\ell_{2}, \ldots, \ell_{\alpha}$ from $L$, but not $\ell_{1}$ itself. We need to replace $\ell_{1}$ in other pointers' lists with locations that are guaranteed to include the value originally stored at $\ell_{1}$ and update the locations' tags accordingly. Thus, for each pointer $\mathrm{p}^{\prime}$ that stores $\ell_{1}$ in its list $L^{\prime}$, we retrieve $\ell_{1}$ 's position pos in $L^{\prime}$ and its corresponding tag $t_{\text {pos }}^{\prime}$. We then replace $\ell_{1}$ in $L^{\prime}$ with $\left\{\ell_{2}, \ldots, \ell_{\alpha}\right\}$ and $t_{\text {pos }}^{\prime}$ in $T^{\prime}$ with $\left\{\left[t_{\text {pos }}^{\prime}\right] \wedge\left[t_{2}\right], \ldots,\left[t_{\text {pos }}^{\prime}\right] \wedge\left[t_{\alpha}\right]\right\}$. If any of $\ell_{i}$ for $i=2, \ldots, \alpha$ already appears in $L^{\prime}$, then that location is not included the second time and its tag is set to the sum of the tag already present in $T^{\prime}$ for location $\ell_{i}$ and $\left[t_{\text {pos }}^{\prime}\right] \wedge\left[t_{i}\right]$.

Returning to our example with $\mathrm{p} 1$ and $\mathrm{p} 2$, we have that prior to calling pfree $(\mathrm{p} 1), \mathrm{p} 1$ stores $\alpha_{1}=2, L_{1}=\left(\ell_{1}, \ell_{2}\right), T_{1}=\left(t_{1}, t_{2}\right)$, and p2 stores $\alpha_{2}=2, L_{2}=\left(\ell_{2}, \ell_{1}\right), T_{2}=\left(t_{1}^{\prime}, t_{2}^{\prime}\right)$. Then, either $t_{1}=$ $t_{1}^{\prime}=1$ and $t_{2}=t_{2}^{\prime}=0$ or $t_{1}=t_{1}^{\prime}=0$ and $t_{2}=t_{2}^{\prime}=1$. Once pfree $(\mathrm{p} 1)$ is called, $\ell_{1}$ is scheduled for deallocation. If $t_{1}=1$, then no changes take place; otherwise $\left(t_{2}=1\right)$, the data from location $\ell_{1}$ is

\footnotetext{
${ }^{3}$ In the event that data stored at the locations contained in the pointer have different sizes, the location with the data of the smallest size should be chosen instead.

${ }^{4}$ Although in the current discussion we assume $p$ is a private pointer that points to a simple non-pointer data type, the same idea will apply when $\mathrm{p}$ points to a pointer or struct. In particular, if $\mathrm{p}$ points to a pointer, the procedure will include merging the lists of pointers stored at locations $\ell_{1}$ and $\ell_{i}$ and updating the tags similar to the formula for simple data types. Furthermore, when $\mathrm{p}$ is a pointer to a struct, each field is updated separately according to its type.
} 
copied into location $\ell_{2}$. We obtain that location $\ell_{1}$ is being removed from $L^{\prime}$ (and the corresponding tag $t_{2}^{\prime}$ from $T^{\prime}$ ) and location $\ell_{2}$ is being added to $L^{\prime}$ with the corresponding tag $t_{2}^{\prime} \wedge t_{2}$. Because $\ell_{2}$ is already present in $L^{\prime}$, it is stored once and the tag becomes $t_{1}^{\prime} \vee\left(t_{2}^{\prime} \wedge t_{2}\right)$. Thus, $L^{\prime}$ now stores a single location and the tag is 1 for any possible set of original tags.

Note that the second step of updating pointers that store location $\ell_{1}$ in their lists is more complex when the pointer $\mathrm{p}$ being freed points to a struct or an array. In those cases, multiple addresses are processed in this step $\left(\ell_{1}\right.$ and other valid addresses that store data at fixed offsets from $\left.\ell_{1}\right)$ depending on the type of data to which $p$ points. All of the operations, however, can be carried out in parallel (as, for instance, is the case in our single-round implementation of calls to pfree).

If the user program is written correctly (i.e., does not leave dangling pointers after a call to free), then our design of pfree will maintain that for each pointer exactly one location's tag is set to 1 and all other locations' tags are set to 0 . When, however, a call to deallocate memory corresponding to a pointer results in dangling pointers, all tags in such pointers can be 0 . For that reason, if a call to pfree causes the number of addresses for some pointer to reduce to 1 , we do not treat the corresponding tag as public. That is, when a program is not correctly written, opening the value of the tag may reveal private information, while assuming that the tag is 1 may modify the program's behavior. Thus, our design maintains privacy even in the presence of programming errors that result in dangling pointers.

We also note that the use of pmalloc or pfree will not be allowed inside conditional statements with private conditions, because these functions have public side effects.

\section{POINTER IMPLEMENTATION IN PICCO}

The previous sections discuss our approach for implementing pointers to private data and related operations, while in this section, we discuss specifics of integrating the approach with PICCO.

\subsection{Specifics of Pointer Implementation in PICCO}

Recall that each pointer starts with a single location (i.e., $\alpha=1$ ) at the time of its declaration, and the list and the number of locations $\alpha$ are updated during pointer assignments. In PICCO, we maintain this information only during program execution and thus the locations that a pointer might store or their number is not necessarily known at compile time.

Also, because PICCO examines the list of variables modified inside the body of each conditional statement with a private condition and updates them differently from when the modification is not surrounded by a private condition, in the case of pointers, we specify how pointers need to be updated inside such statements using Algorithm 1 and the compiler will process all variables inside the body of conditional statements with private conditions.

Optimizations. In PICCO that uses linear secret sharing, performance of pointer dereferencing with indirection level 1 is low, because this operation is implemented as an inner product that costs only one interactive operation (recall that we replace the OR with a sum, because at most one tag is set to 1). Similarly, the computation for pointer dereferencing with higher indirection level can be replaced with the sum, because at most one tag can be set. In addition, evaluating predicates over pointers when the result of the evaluation is private can be optimized for certain types of predicates. For example, in the case of pointer equality, the general solution is to compute $\left[\ell_{1}\right]=$ $\sum_{\ell_{i}^{(1)} \in L_{1}} \ell_{i}^{(1)}\left[t_{i}^{(1)}\right]$ and $\left[\ell_{2}\right]=\sum_{\ell_{i}^{(2)} \in L_{2}} \ell_{i}^{(2)}\left[t_{i}^{(2)}\right]$ and then compare $\left[\ell_{1}\right]$ and $\left[\ell_{2}\right]$ for equality, while an optimized implementation computes $\sum_{\ell_{i}^{(1)} \in L_{1} \cap L_{2}}\left[t_{i}^{(1)}\right]\left[t_{j}^{(2)}\right]$, where $\ell_{i}^{(1)}=\ell_{j}^{(2)}$ for each $\ell_{i}^{(1)} \in L_{1} \cap$ $L_{2}$. The latter can be implemented as the inner product that costs a single interactive operation and is significantly lower than the cost of comparing two private integers. 
Pointer predicates. Recall that with pointer predicates some checking needs to be deferred to run time. In particular, if pointers to private data are used in a predicate to form a conditional statement and the result of its evaluation is private, the usual constraints for the body of conditional statements with private conditions apply. We address this by evaluating the body of such conditional statements for public side effects at compile time (as in the original PICCO design). If the body contains public side effects, then the transformed program will include checks for the status of the conditional statement at run time. If the result of evaluating the conditional statement is determined to be private at run time (and public side effects are present in the body of the statement), then execution will be aborted with an error due to a possible information leak.

Note that the fact whether the execution is aborted or not never leaks private information, as this decision is solely based on public data. That is, an abort takes place when (i) the result of evaluating a predicate on two or more pointers is treated as private and (ii) the body of the conditional statement that uses the predicate contains public side effects. Whether the result of evaluating the predicate is public or not is public knowledge, because it is determined by the public locations stored in the pointers and the predicate itself. Similarly, whether the body of the conditional statement has public side effects or not is based on the public code that forms the body of the conditional statement.

Pointer casting. Because in PICCO simple data types can be defined to have any bitlength, casting, for example, a pointer of one integer type to a pointer of another integer type does not guarantee that one data type will have a bitlength multiple of another. In that case, we still calculate what the relevant portion of the memory is based on the position of the memory being dereferenced, but the last, partially filled, element might not be reliably extracted. For example, suppose some memory was filled as a 3-element 30-bit integer array. When it is cast to an array of 20-bit integers, the fourth elements will be extracted as bits 61-80 of the original data, while retrieving the fifth element might result in memory violation, because there is not enough data in the original array to fully form that element.

\subsection{Other Optimizations}

Because the computation involved in computing a pointer's dereferenced value is interactive (and thus is relatively expensive) when the pointer stores multiple locations, we considered caching and reusing its result. That is, when a pointer's dereferenced value is computed, we can store it in the pointer structure and reuse the value on consecutive dereference operations when there are no changes to the values stored at all pointer's locations $L$ between such operations. Once any value stored at one of the pointer's locations is modified, the cached dereferenced value needs to be marked as out of date. A similar caching technique can also be applied to the computation on private tags that takes place during pointer update and dereference operations. Note that private tags can be viewed as aggregation of conjunctions of 1-bit private variables that denote the evaluation results of private conditions (or their negations) in user programs. Because, once computed, the variables will have fixed values, the conjunction results of those variables can be cached in our framework in a lookup table and allow for their retrieval when the same conjunctions need to be repeatedly computed. This optimization will result in considerable savings when multiple private pointers are updated or dereferenced within the body of a conditional statement with a private condition. The savings due to either of the above caching optimizations are, however, applicationdependent and require additional program analysis at program parse and translation time. Thus, these optimizations are not presently a part of our implementation.

Another possible optimization can lower a program's memory footprint by reusing data structures created for pointers to private data. In particular, when a pointer is assigned another pointer's value (as in $p=p 1$ ), we could have both pointers pointing to the same data structure instead of 
creating its copy. When, however, one of the pointers with the shared data structure is being modified, it should be unlinked from the shared data structure and its data structure modified accordingly. Implementing this would require that each pointer data structure is associated with a list of pointer variables that are using it. Furthermore, a data structure can be reused only when all information stored in it is identical for multiple pointers (i.e., not only the locations in $L$, but also the private tags in $T$ ). Because different pointers often have distinct roles in user programs, the expected savings are not very large. This optimization is presently not a part of our implementation.

\subsection{Pointers as Arrays with Known Bounds}

In PICCO, statically allocated arrays of private variables have the array size stored with them (which is known at the array creation time). Knowing the size of the arrays allows the compiler to support a number of important operations on arrays. Most significantly, this permits the use of private indexing with arrays, when an element at a private position $i$ is retrieved from an array a using syntax $\mathrm{a}[\mathrm{i}]$. (The size of the array must be known to support private indexing, regardless of what technique is used to implement it.) This also permits the use of other operations such as inner (or dot) products on two arrays, which were introduced to optimize runtime of compiled programs.

We treat private indexing as an essential part of secure computation with private data and would like to see it supported for arrays dynamically allocated on the heap. This means that we would like to offer pointer indexing $p[i]$ with private $i$ and private pointer $p$. The main challenge that we need to overcome is the fact that the size of the memory pointed by $\mathrm{p}$ is not available in $\mathrm{C}$. Furthermore, a location stored in $\mathrm{p}$ may be arbitrary and do not correspond to a valid memory address (i.e., be unaccessible by the program, correspond to memory marked as not being in use or any location from the program's stack, etc.). This means that a pointer can take on many addresses that were not allocated for variable use and for which the corresponding size cannot be meaningfully determined (i.e., accessing such addresses would trigger invalid memory access exceptions in safe programming languages). The size of properly allocated memory, however, can be determined and utilized to implement private indexing (and other operations that require array size) with pointers to private data. In particular, all memory that malloc allocates on the heap is marked with the size of each allocated block. Thus, we can use the information that malloc/free maintain to determine whether a pointer content falls within a properly allocated memory block, and if it is the case, access the block's size and use it to implement private indexing.

In more detail, in addition to using private indexing with statically allocated arrays (as already implemented in PICCO), we permit private indexing to be used with pointers to private data. The latter is only successful if the location stored in the pointer ${ }^{5}$ was allocated via a prior call to pmalloc (and it was not deallocated during a call to pfree). Because the secure implementation that PICCO produces makes calls to malloc for various internal tasks, as opposed to only user-triggered calls to pmalloc, there is a need to maintain address ranges corresponding to userallocated dynamic memory. For that reason, the program internally maintains a list of addresses returned by malloc that correspond to memory requested by the user program via calls to pmalloc (and an address is taken off the list if it is being freed). Then, when private indexing $\mathrm{p}[\mathrm{i}]$ is called in the user program and the pointer stores address $\ell$, we iterate through the list of maintained addresses. For each such address $l$, we retrieve the corresponding block size $s$ from the information

\footnotetext{
${ }^{5}$ The current discussion refers to a single location stored in a pointer, which we view as the most common use of private indexing. When the pointer contains multiple locations, the operation is performed on each location separately and the results are combined in the same way as during pointer dereferencing.
} 
stored by malloc and check whether $l \leq \ell<l+s$ and the offset of $\ell$ from $l$ is a multiple of the data type size. If these checks succeed for at least one location on the allocated address list, then $s$ is adjusted for the data type size and is used as the size of the array to which $p$ points. Note that with this implementation $\ell$ does not have to correspond to the beginning of the memory block. Then, when $\ell$ is not the address of the beginning of the array, $i$ can legitimately take negative values.

Under the circumstances when the address $\ell$ does not fall within any memory block dynamically allocated by the user, the private indexing operation is not performed and the returned result is set to be secret-shares of 0 (note that, regardless to what value the result is set in such a case, it is not guaranteed to be interpreted as an error). We thus proceed with the computation despite the error, but send signal SIGBUS ${ }^{6}$ and store an error message in a fixed location, so that the program can catch the signal and act on it. Note that the address that each call to pmalloc returns is always public information and the programmer can avoid using invalid addresses. Ideally, the fact that the private indexing operation cannot be carried out on the given address is determined before the program is run, at compile time. This, however, will not always be possible and for some incorrectly written user programs the error will not be triggered until the program is executed (even programming languages that perform static analysis of user programs do array-bounds checking dynamically). The best we can do is to perform static program analysis at compile time and warn the user about places where such an error might be possible.

\section{ANALYSIS}

After discussing multiple aspects of private pointer design and its uses in programming, in this section we summarize the notion of pointers to private data and operations on it and formally show that program execution that involves pointers to private data complies with a standard definition of security used in secure multi-party computation.

A pointer to private data is defined as a C-style pointer to a private object storing location information of the object, where a private object can be one of the following types:

(1) a primitive private data type (private int, float, etc.);

(2) a composite data type (C-style struct), each element of which is a private object;

(3) a function with no public side effects;

(4) a pointer to a private object.

The second and fourth categories define a pointer to private data recursively, which means that a pointer can have any indirection level, nested struct types, and any combination of primitive, composite, and pointer data types.

The previously defined operations on pointers to private objects are:

(1) Pointer read and update with value $v$, denoted as $\operatorname{read}(\mathrm{p})$ and update( $\mathrm{p}, v)$, respectively.

(2) Dereferenced pointer read and update, denoted as $\operatorname{read}(* p)$ and update $(* p, v)$, respectively.

(3) Pointer update inside a conditional statement with a private condition. Following prior work, we use multiplexor notation to denote this operation as $\operatorname{mux}\left(\mathrm{p}, v_{1}, v_{2}\right.$, cond $)$, where $\mathrm{p}$ is set to $v_{1}$ if private cond evaluates to 1 and to $v_{2}$ otherwise. Pointer read inside a conditional statement with a private condition is processed identically to a conventional $\operatorname{read} \operatorname{read}(p)$ and thus is not listed as a separate operation.

\footnotetext{
${ }^{6}$ Alternatively, custom SIGUSR1 or SIGUSR2 can be triggered if the user program is known not to use it.
} 
(4) Dereferenced pointer update inside a conditional statement with a private condition, which we denote as $\operatorname{mux}\left(* \mathrm{p}, v_{1}, v_{2}\right.$, cond $)$. Similar to the previous case, processing of dereferenced pointer reads is not affected by the presence of conditional statements.

(5) Dynamic memory allocation in the form of malloc; for an assignment $p=\operatorname{pmalloc}(n$, type), we use notation alloc( $\mathrm{p}, n$, type).

(6) Dynamic memory deallocation as in pfree ( $p)$, denoted as dealloc(p).

(7) Array indexing $\mathrm{p}[i]$ with a public index $i$. This is treated as a generalization of pointer dereferencing, and we use notation $\operatorname{read}(\mathrm{p}, i), \operatorname{update}(\mathrm{p}, i, v), \operatorname{mux}\left(\mathrm{p}, i, v_{1}, v_{2}\right.$, cond $)$ to denote read, update, and update inside a conditional statement with a private condition, respectively.

(8) Array indexing $\mathrm{p}[i]$ with a private index $i$ is also divided into three operations $\operatorname{readp}(\mathrm{p}, i)$, $\operatorname{updatep}(\mathrm{p}, i, v)$, and $\operatorname{muxp}\left(\mathrm{p}, i, v_{1}, v_{2}, c o n d\right)$. These operations can only be performed on locations stored in a pointer that correspond to arrays with known bounds (i.e., allocated using the pmalloc interface or static array declaration).

(9) Evaluation of predicate $f$ on one or more pointers $\mathrm{p}_{1}, \ldots$, denoted as $\operatorname{pred}\left(f, \mathrm{p}_{1}, \ldots\right)$.

(10) Pointer casting, denoted as cast(p,type), where type is the data type to which $\mathrm{p}$ is cast.

(11) Pointer addition/subtraction, denoted as $\operatorname{add}(\mathrm{p}, u)$, where value $u$ is possibly negative.

(12) Pointer difference $\operatorname{diff}\left(\mathrm{p}_{1}, \mathrm{p}_{2}\right)$.

In the above, $v, v_{1}$, and $v_{2}$ correspond to either values associated with private objects or data structures corresponding to pointers to private objects, depending on the context. The value of cond is always a private bit, while variables $n, i, u$, and type are public. Any variable can be read inside a conditional statement with a private condition, but updates can be performed only as specified using the mux operations.

This list implicitly defines operations that cannot be performed on pointers to private objects and will be rejected by the compiler. That is, addresses of public objects cannot be used to update a pointer to private data (either via update or mux); a pointer to public data or a mix of private and private fields defined with a struct construct cannot be modified inside a conditional statement with a private condition (i.e., there is no corresponding mux operation); pmalloc and pfree cannot be called inside conditional statements with private conditions (i.e., there is no mux operations for them); last, casting cannot be called inside conditional statements with private conditions as it modifies publicly stored data.

Recall that implementation of pointers maintains the invariant that in a well-formed program there is exactly one true location associated with a private object. The invariant may be violated only when memory associated with a pointer is being deallocated when the pointer is still in use (in such a case, no tag is set to 1 and dereferencing the pointer will return no data).

In showing security of pointer-related operations that reference private data, we use a traditional simulation-based definition of security. Because PICCO is built on top of $(n, t)$-threshold secret sharing techniques with $n \geq 3$ computational parties, we utilize the same setup in our security analysis. Similarly, because the underlying techniques offer information theoretic security, we utilize statistical (as opposed to computational) indistinguishability in the security definition.

Definition 7.1. Let parties $p_{1}, \ldots, p_{n}$ engage in a protocol $\Pi$ that evaluates program $P$ on a mix of public and private data. Let $\operatorname{VIEW}_{\Pi}\left(p_{i}\right)$ denote the view of $p_{i}$ during the execution of $\Pi$, which is formed by its input, internal random coin tosses $r_{i}$, and messages $m_{1}, \ldots, m_{k}$ passed between the parties during protocol execution: $\operatorname{VIEW}_{\Pi}\left(p_{i}\right)=\left(i n_{i}, r_{i}, m_{1}, \ldots, m_{k}\right)$. Let $I$ denote a subset of the participants of size $t$ and $\operatorname{VIEW}_{\Pi}(I)$ denote the combined view of the participants in $I$ during the execution of $\Pi$. Protocol $\Pi$ is said to be $t$-private in the presence of semi-honest adversaries if for each coalition of size at most $t<n / 2$ there exists a probabilistic polynomial time simulator 
$S_{I}$ that given the input of the parties in $I, P$, and $P$ 's output, produces a view statistically indistinguishable from $\operatorname{VIEW}_{\Pi}(I)$ together with the output of the parties in $I$.

Theorem 7.2. Any program $P$ with pointers to public and private data and no out-of-boundary array access compiled by PICCO is translated into a $t$-private protocol for any $t<n / 2$ when the computation is carried out by $n$ parties.

The proof of Theorem 7.2 is provided in the full version [25]. We note that our security result above is stated for programs that PICCO successfully compiles and that do not contain out of boundary array accesses. As previously discussed, the compiler will reject almost all types of improperly written programs during static analysis at compilation time. For example, if a pointer to public data is modified inside a conditional statement with a private condition, the program will not compile. This means that the compiler will filter out the majority of programs with errors, while any well-formed program enjoys simulatable security.

The main type of errors that the compiler may not be able to detect during static analysis deals with memory access to invalid locations (e.g., out of boundary array access, access to a hard-coded invalid location, etc.). When such accesses are triggered, one or more computational parties might not be able to continue with the execution and quit on error, but no privacy violations take place. This is because while incorrect shares of private data might be read, the data will still remain in the protected form and cannot be reconstructed without the programmer's original intent. Note that this discussion applies only to dereferencing a pointer with no offset (e.g., as in $\star p)$ or with a publicly known offset (e.g., as in $\mathrm{p}[i]$ with public $i$ ), because in our implementation accessing an array at a private location never results in out of boundary access (i.e., calling this operation on an improperly allocated array will be skipped at runtime).

Because the memory layout may differ on different platforms, illegal memory accesses might result in different behavior of the computational parties (e.g., execution at one party might result in a segmentation fault faster than at another party). As our simulator is not guaranteed to run in an identical environment to those of the honest parties, the simulation might be distinguishable for programs with illegal memory accesses based on the point of execution when any given party aborts the computation. Thus, we exclude such programs from the security statement of Theorem 7.2, but still guarantee that any program that compiles in our framework will not result in privacy violations.

To summarize, our solution guarantees that any program that can be compiled in our framework never reveals any unauthorized information about private data. Simulation of a poorly written program with illegal memory accesses may not be indistinguishable from its real execution, but any properly written program enjoys simulation-based security.

\section{POINTER-BASED DATA STRUCTURES}

There are several popular data structures typically built using pointers. In this section, we discuss how they would be implemented using pointers to private data and in what complexities their performance results. In particular, we explore linked lists, trees, stacks, and queues.

\subsection{Linked Lists}

A linked list consists of a sequentially linked group of nodes. For a singly linked list, each node is composed of data and a reference in the form of a pointer to the next node in the sequence, while for more complex variants such as doubly and circular linked lists the reference field incorporates additional links. A linked list allows for efficient node insertion and removal, which makes it an ideal candidate for implementation of stacks and queues as well as representation of graphs that uses an adjacency list. In what follows, we discuss implementations of linked lists that 
store private data. We start by analyzing various operations in standard linked lists and then elaborate on the special case when a linked list stores sorted data. The latter does not represent a typical use of linked lists in programming (and does not even have attractive features) but is provided as a relatively simple way to demonstrate what form working with sorted data can take in a secure computation framework.

Throughout this section, we express complexities of the operations as a function of $n$, where $n$ is the "visible" list size. This value will correspond to the actual list size when delete operations remove an element from the list so that its size is reduced, while it will correspond to the number of insertions (i.e., the maximum list size) when delete operations only mark data as deleted (to hide whether the element was found or not) but not reduce the list size. The same notation applied to other data structures discussed in this section when insertions/deletions are based on the result of private conditions.

Standard linked lists. Because of ubiquitous use of linked lists in programming, we analyze different possible uses of linked lists and the corresponding operations. When a linked list stores public data, node insertion has cost $O(1)$ as a node is inserted in a fixed place (e.g., beginning or end of the list). Performing a search requires $O(n)$ time, where $n$ is the number of nodes in the list, because the nodes are traversed sequentially. Deleting a node from a fixed place (i.e., beginning or end of the list as done in the case of stacks and queues) involves $O(1)$ time, but when deletion is preceded by a search (and the found node is deleted), the search together with deletion require $O(n)$ time.

When a linked list stores private data, the reference field holds a pointer to private data (i.e., a record of the same type) and at the time of node creation, the pointer stores a single location. Without loss of generality, let nodes be inserted in the beginning of the list. Node insertion then places a new node in the beginning of the list manipulating pointers as before, which still takes $O(1)$ time and is very efficient. Searching a list involves $n$ private comparisons and all nodes need to be processed as not to reveal the result of individual comparisons on private data, and the total work is $O(n)$. Similarly, when a node is deleted from the beginning (or end) of the list, the time complexity is $O(1)$ and each node's pointer still stores a single location. It is only when nodes need to be removed from varying positions in the list and the position itself needs to be protected, pointers can start acquiring multiple locations, which causes the time complexity of list traversal and deletion after a search to go up. However, when the fact whether the searched data was found in the list or not must remain private, we cannot remove any node, but instead need to erase the content of a found node (if present) with a value that indicates "no data." In this case, all pointers still contain a single location and the cost of list search and other operations do not change, but the list will never reduce in its size. We defer the discussion of the case when the node is guaranteed to be found in a search and needs to be removed from a private location until the end of this subsection.

Sorted linked lists. As mentioned before, we discuss sorted linked lists only as a means of demonstrating how sorted data might be processed using a general-purpose secure computation compiler. It should be understood that this is not a typical use of linked lists or even not the best way of working with sorted data. We use the results of this discussion in our consecutive description.

Now when a node is being inserted in a linked list, the insertion position must be determined based on the data stored in the list, which involves $O(n)$ time with public data (and the complexities of other operations are the same as before). When we work with private data, the location where the node is being inserted must remain private (since it depends on private data) and the execution needs to simulate node insertion at every possible position. Consider the following two ways of inserting a node and the performance in which they result: 
(1) Pointer updates: The first is a traditional implementation of node insertion in a linked list, where if the correct insertion point is found, we update the pointer of the found node in the list to point to the new node and the pointer of the new node to point to the next node in the list. Because this conditional statement is based on private data, this will result in adding one location to the pointer in the found node and one location to the pointer in the node being inserted. After executing this operation for every node of the list, the pointer of each node in the original list stores 2 locations and the pointer in the newly inserted node stores $n$ locations. When this operation is performed repeatedly, each node in the list acquires more and more locations (to the maximum of the current list size). This means that if the list is built by inserting one node at a time, the cost of node insertion and list traversal becomes $O\left(n^{2}\right)$. Node deletion after a search also takes $O\left(n^{2}\right)$ time, while node deletion from a fixed location is bounded by $O(n)$. When, however, only a constant number of nodes are inserted into an existing list (which, e.g., can be provided as sorted input into the program), the complexity of all operations are unchanged from the public data case.

(2) Data updates: Another possible implementation of sorted linked lists is to always insert a new node at the beginning of the list and keep swapping its content with the next node on the list until the correct insertion point is found. When this algorithm is implemented obliviously on private data using an SMC compiler, the computation processes each node on the list starting with the newly inserted node and based on the result of private comparison of current and new data either performs the swap or keeps the data unchanged. After each node insertion the reference field of each node still points to a single node in the list and therefore the complexity of all operations are unchanged from their public versions.

Thus, it is clear that we want to avoid acquiring a large number of locations in each reference field of a pointer-based data structure and privately moving data (as opposed to privately moving pointers) is preferred when working with sorted data.

Node deletion in standard linked lists. We can now return to the question of deleting a node from a private location in a standard (unsorted) linked list when it is known that the searched node is present and needs to be removed from the list. The above two approaches of inserting a node in a private position also apply to deleting a node from a private position. The first, standard, approach of manipulating pointers will result in acquiring multiple locations at each pointer, which degrades performance of all operations. Using the second approach of data updates, we can obliviously place the data to be deleted into the first node on the list (after scanning the nodes and swapping values based on private data comparisons) and then simply remove it from the list. This will maintain optimal complexities of all operations. The above tells us that traditional implementations of data structures can exhibit performance substantially worse than alternative implementations in a secure computation framework and our analysis can be viewed as a step in making informed decisions about implementation needs.

\subsection{Trees}

Trees implement hierarchical data structures commonly used to store sorted data and make searching it easy. A tree node is typically comprised of data and a list of references to its child nodes. In an $n$-node balanced search tree, all of searching, node insertion, and node deletion take $O(\log n)$ time. Unfortunately, these complexities greatly change when we write a program to implement a search tree on private data. In what follows, we distinguish between trees that are 
pre-built using the information available prior to the start of the computation and trees built gradually using information that becomes available as the computation proceeds:

(1) Pre-built trees. Consider a balanced binary search tree and suppose that we want to perform a search on the tree. A traditional implementation involves $O(\log n)$ conditional statements to traverse the tree from the root to a leaf choosing either the left or right child of the current node. When the data is private, such statements use private conditions and thus both branches of the computation must be executed. The result is that the sequence of $O(\log n)$ nested private conditions results in executing all possible $O(n)$ branches of the computation and touches all nodes in the tree. This is an exponential increase in the complexity compared to working with public data, even if we do not consider node insertions and deletions that result in node rotations to balance the tree (which are discussed next together with gradually-built trees).

(2) Gradually-built trees. By analogy with inserting nodes into a sorted linked list, we can either manipulate pointers to insert a new node at the appropriate place in the tree or insert the node in a fixed location and move the data in place. The complexity of the latter option is $O(n)$ for insertions, deletions, and search, and we take a closer look at the former. As we traverse the tree looking for the place to insert the new node, similar to searching, all nodes will be touched (as a result of nested private conditions). Furthermore, because the execution cannot reveal the place into which the new node is inserted, pointers in all nodes will acquire new locations. If we add computation associated with node rotations when the tree becomes unbalanced, then pointers will be acquiring new locations even faster (to the maximum of $n-1$ per pointer). After repeatedly calling insert to gradually build the tree, eventually each node will point to all other nodes resulting in $O\left(n^{2}\right)$ complexity for insertions, deletions, and searching. Such complexity is clearly avoidable, and alternative implementations should be pursued.

Search trees represent the worst possible scenario where implementing an algorithm on private data using a general-purpose compiler incurs an exponential increase in its runtime compared to the public data counterpart. As is evident from our discussion of linked lists and trees, searching an $n$-element store for a single element cannot be performed in less than linear time using generic techniques, regardless of whether the data is stored sorted or not. It means that without custom, internally built implementations of specific data structures it is conceptually simpler and more efficient to maintain data in unsorted form, use append for insertion $(O(1)$ time), and shift data to implement deletion.

\subsection{Stacks}

A stack is characterized by the last-in, first-out (LIFO) behavior, which is achieved using push and pop operations. It has several fundamental applications such as parsing expressions (e.g., parsing programs in compilers), backtracking, and implementing function calls within an executable program. Our analysis and consecutive implementation of stack that works with private data demonstrate its appeal for secure computation.

A pointer-based implementation of a stack is built using a linked list, where a node is always inserted at the head of the list and is always removed from the head as well, either of which takes $O(1)$ time. As was discussed in Section 8.1, implementing these operations on private data maintains constant time complexities.

When using a stack with private data, we also consider the possibility that push and pop operations might be performed inside conditional statements with private conditions, in which case it is not publicly known whether the operation takes place and what record might be on top of the 
stack. Then, if we implement a conditional private push operation by manipulating pointers, the top of the stack will store $m+1$ locations when the last $m$ push operations were based on private conditions. Implementing a push operation is then equivalent to executing the code:

1. node $\mathrm{p}=$ new node () ;

2. if (priv-cond)

3. $\mathrm{p}->$ next $=$ top;

4. top $=\mathrm{p}$;

Because both $\mathrm{p}$ and $\mathrm{p}->$ next store only a single location at the time of conditional push, merging the lists of $\mathrm{p}^{->}$next and top takes $O(m)$ time. Similarly, merging the lists of top and p takes $O(m)$ time.

Implementing a pop operation within a private condition involves executing code:

1. if (priv-cond)

2. temp $=$ top;

3. top $=$ top->next;

4. // use temp

The complexity of this operation is dominated by the second assignment. Because top points to $O(m)$ locations, and the next field of each of its locations can store $O(m)$ locations as well, the overall complexity of that assignment is $O\left(\mathrm{~m}^{2}\right)$. This means that the worst time complexity of a conditional push is $O(n)$ for a stack containing $n$ records and it is $O\left(n^{2}\right)$ for a conditional pop.

If we instead implement push and pop operations that depend on private conditions by maintaining a single chain of records (with pointers containing a single location) and data update, then push and pop operations result in $O(1)$ and $O(n)$ work, respectively. That is, we can always insert a new node (with data or no data depending on the private condition) into the stack and take $O(n)$ time during pop to privately locate the first node with data (and erase the data as necessary).

\subsection{Queues}

Queue is another important data structure used to maintain a set of entities or events in a specified order that are waiting to be served. We can distinguish between first-in, first-out (FIFO), last-in, first-out (LIFO), and priority queues. Implementing a queue involves maintaining two pointers: the head and the tail. The head points to the beginning of the queue, i.e., the element that will be removed by a dequeue operation, and the tail points to the last element added to the queue using an enqueue operation.

Similar to the stack, when enqueue and dequeue operations in a FIFO queue are implemented on public data or private data outside of private conditional statements, their complexities are $O(1)$. Their complexities for enqueue and dequeue operations are also $O(n)$ and $O\left(n^{2}\right)$, respectively, when implemented through private pointer manipulation (the implementation needs to maintain two pointers for the head and tail of the queue, but updating the second pointer does not asymptotically increase the amount of work) and $O(1)$ and $O(n)$, respectively, when private data update is used.

In a priority queue, each node additionally stores priority (which we assume is private) and dequeue removes a node with the highest priority. The complexity of priority queue operations depends on the underlying data structure used to implement it. The best-known complexities for public data are $O(\log n)$ for enqueue $(O(1)$ average case) and $O(\log n)$ for dequeue using a heap.

Suppose first that there are no conditional statements with private conditions. If we use a linked list to store queue nodes, then we can achieve $O(1)$ for enqueue and $O(n)$ for dequeue (i.e., always store a newly inserted node in the beginning and remove the highest priority node from a private location as a result of the search for the highest priority element) or $O(n)$ for enqueue and $O(1)$ for 
Table 1. Performance of Various Data Structures Using Pointers to Private Data

\begin{tabular}{|l|c|c|c|}
\hline Data structure & Insert & Delete & Search \\
\hline Linked list & $O(1)$ & $O(1)$ & $O(n)$ \\
\hline Linked list (delete at private location) & $O(1)$ & $O(n)$ & $O(n)$ \\
\hline Search tree & $O(n)$ & $O(n)$ & $O(n)$ \\
\hline Stack or queue & $O(1)$ & $O(1)$ & - \\
\hline $\begin{array}{l}\text { Stack (conditional private push \& pop) or } \\
\text { queue (conditional private enqueue \& dequeue) }\end{array}$ & $O(1)$ & $O(n)$ & - \\
\hline Priority queue & $O(1)$ & $O(n)$ & - \\
\hline Priority queue (conditional private enqueue \& dequeue) & $O(n)$ & $O(1)$ & - \\
\hline
\end{tabular}

dequeue (i.e., store the list sorted and always remove the first node during dequeue). Then, if the operations depend on private conditions, we can maintain $O(1)$ for enqueue and $O(n)$ for dequeue using a very similar approach to that of regular queues and stacks. That is, we always insert an element into the beginning of the queue as a result of a conditional enqueue (if the condition is false, the element is empty), and during dequeue, we scan the queue for the highest priority element and erase it from the queue if the condition is true.

If the underlying implementation is a heap, then we insert a new node in a fixed leaf location and use $O(\log n)$ compare-and-exchange operations to maintain the invariant of a max-heap to implement enqueue. Realizing dequeue, however, requires $O(n)$ work, because it cannot be revealed what path was traversed from the root to a leaf (since the path choice depends on private priorities). Similar to other implementations, we can maintain these complexities even when enqueue and dequeue are performed as a result of private condition evaluation.

\subsection{Summary}

Before we conclude this section, we will summarize performance of different data structures that can be implemented on private data using newly introduced pointers to private data or records. Table 1 lists the best performance we could achieve using a pointer-based implementation of the data structures discussed in this section. Priority queue has two alternative implementations. Recall that in all data structures with conditional operations performance depends on the number of insertions as opposed to the actual data size. We note that the complexities in Table 1 can be directly linked to the amount of memory consumed by those data structures, with small fixed constants hidden behind the asymptotic notation.

These data structures can also be evaluated using alternative mechanisms. For example, our analysis suggests that implementing these data structures using arrays of private data instead of pointers to private data would result in the same complexities (which is often the case for public data as well). Also, utilizing ORAM-based implementation can improve asymptotic complexity of some (but not all) data structures and can lead to faster runtime in practice at least for large enough data sets. The most pronounced benefit of using ORAM will be observed for implementing search trees, where all operations can be performed in polylogarithmic (in $n$ ) time (e.g., using the solution in Reference [23]). On the other hand, using ORAM for linked lists can only increase the complexity of its operations (even the complexity of a delete at a private location following a search cannot be reduced below $O(n)$ ). Other data structures that can benefit from ORAM-based implementations are stacks and queues where the operations that update the data structures are performed inside private conditional statements. ORAM techniques, however, involve larger constants 
behind the big-O notation than simple operations and their initial setup cost is also significant. We thus leave a thorough comparison of ORAM vs. pointer or array based implementations of various data structures in this framework as a direction of future work.

\section{PERFORMANCE EVALUATION}

In this section, we report on the results of our implementation and evaluation of a number of representative programs that utilize pointers to private data. Because such programs have not been previously evaluated in the context of secure multi-party computation, we cannot draw comparisons with prior work. In some cases, however, we are able to measure the cost of using pointers, or the cost of a pointer-based data structure, in a program by implementing the same or reduced functionality that makes no use of pointers. Note that because PICCO can be used for both secure multi-party computation and outsourcing, the inputs in these programs can come from one or more input parties/clients. The programs that we implemented and evaluated as part of this work are:

(1) The first program constructs a linked list from private data read from the input and then traverses the list to count the number of times a particular data value appears in the list. This is a traditional implementation of a linked list, where each record with private data is prepended to the beginning of the list when building it. Because of space constraints, the program is given in the full version [25] (Figure 1).

We next notice that this program is sub-optimal in terms of its run time, because it does not utilize concurrent execution capabilities provided in PICCO. For that reason, we also implement an optimized version of this program. The difference is that all private comparisons during the list traversal are executed in a single round using PICCO's batch constructs.

(2) To evaluate pointer-based implementations that work with private data maintained in a sorted form, and more generally privately manipulating pointer locations vs. obliviously moving data, we build a program for a sorted linked list. The functionality of this program is similar to that of the first program (i.e., create a linked list and then traverse it to count the number of occurrences of a given data item in it) and the difference is in the way the list is build. We evaluate two variants of the program corresponding to pointer update (PU) and data update (DU) as described in Section 8.1. The programs for the DU and PU variants are provided in Reference [25] (Figures 2 and 3).

(3) The third program implements mergesort that takes an array of unsorted integers as its input. The program makes an extensive use of pointers to private data to pass data by reference to a function that conditionally swaps two data items based on their values (i.e., performs the so-called compare-and-exchange operations). Mergesort was chosen not necessarily because it provides the best performance for an oblivious sort, the objective instead was to demonstrate how performance of a program that utilizes pointers to private data (and exercises modular design of a program) compares to a similar program that does not use pointers. We thus also evaluate another version of mergesort that performs compare-and-exchange operations in place (without calling any function) and makes no use of pointers. The pointer-based and non-pointer-based implementations of mergesort are available in Reference [25] (Figures 4 and 5).

Note that for this program it is straightforward to eliminate the use of pointers (which are are used for passing data by reference) while preserving the program functionality. Comparison to alternative, non-pointer-based implementations for other functionalities (for instance, for data structures such as linked lists and a shift-reduce parser functionality below) is deferred to the end of this section. 
(4) Our last program implements a shift-reduce parser for a context-free grammar (CFG) on private data. This is one of the fundamental applications that can now be naturally implemented using the compiler by building and maintaining a stack, once support for pointers to private data is in place. We choose a CFG that corresponds to algebraic expressions consisting of additions, multiplications, and parentheses on private integer variables, which is specified as follows:

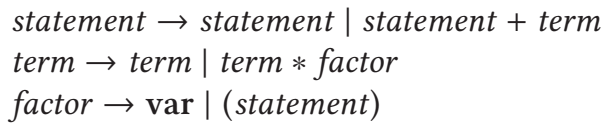

Here, all variables are shown in italics, while terminals are set in bold font. The grammar can obviously be generalized to more complex expressions and programs that work with private as well as public variables of different types. We view this application as enabling one to evaluate a custom function on private data without writing and compiling a separate program for each function. That is, both the function to be evaluated and its input (consisting of private data) are provided as input to the parser. We note that it is possible for the function or the grammar rules to be private as well, but this would result in an increase in the program performance. Our parser uses one lookahead character, and due to the complexity of the implementation, the program itself is not included in the article.

To approximate performance overhead associated with using a pointer-based stack, we create a program that performs only arithmetic operations on private data that are given to the parser and that the parser executes. Note that unlike evaluation of mergesort, these are not equivalent functionalities. That is, one program is much more complex, parses its input according to the CF grammar, maintains a stack, and so on, while the other only performs additions and multiplications.

Note that most of these programs already exercise dynamic memory allocation (i.e., all linked list programs and the shift-reduce parser). However, to provide a more complete evaluation of dynamic memory management, we also include experiments that measure the overhead of dynamic memory deallocation. Thus, we incorporate calls to pfree to two programs: (i) we call pfree as part of the shift-reduce parser at the end of each pop operation, and (ii) we evaluate the cost of removing the head node in a sorted linked list built using pointer update (Figure 3 in Reference [25]). These were chosen as natural applications of memory deallocation, where pointers to private objects contain a single and multiple locations, respectively. In the second case, the head stores locations of all nodes on the list and the overhead of pfree includes updating the structures of other pointers on the list upon memory deallocation.

Each program was compiled using PICCO, extended with pointer support as described in this work, and run in a distributed setting with three computational parties. All compiled programs utilize the GMP library for large number arithmetic and OpenSSL to implement secure channels between each pair of computational parties. We ran all of our experiments using three $2.4 \mathrm{GHz}$ 6-core machines running Red Hat Linux and connected through $1 \mathrm{~Gb} / \mathrm{s}$ Ethernet. Each experiment was run 10 times, and we report the mean time over all runs and the corresponding deviation from the mean observed in the experiments. The results of the experiments for working with unsorted and sorted data are given in Tables 2 and 3, respectively.

As can be seen from the tables, each program was run on data of different sizes. For all linked lists programs as well as mergesort, the data size corresponds to the number of elements in the input set, while for the shift-reduce parser and arithmetic operations the size corresponds to the number of arithmetic operations in the formula, which were a mix of $90 \%$ multiplications and $10 \%$ 
Table 2. Performance of Representative Programs with Unsorted Data Structures Measured in Seconds

\begin{tabular}{|l|c|c|c|c|c|c|c|}
\hline \multirow{2}{*}{ Program } & Field & \multicolumn{5}{|c|}{ Data size } \\
\cline { 3 - 8 } & size & $2^{5}$ & $2^{8}$ & $2^{11}$ & $2^{14}$ & $2^{17}$ & $2^{20}$ \\
\hline \hline \multirow{2}{*}{$\begin{array}{l}\text { Linked list } \\
\text { (list building, traversal, } \\
\text { and optimized traversal) }\end{array}$} & \multirow{3}{*}{81} & $0.0004 \pm 5 \%$ & $0.003 \pm 4 \%$ & $0.014 \pm 3 \%$ & $0.097 \pm 2 \%$ & $0.760 \pm 1 \%$ & $5.401 \pm 1 \%$ \\
\cline { 3 - 8 } & & $0.086 \pm 1 \%$ & $0.661 \pm 1 \%$ & $5.302 \pm 3 \%$ & $42.27 \pm 1 \%$ & $337.8 \pm 1 \%$ & $2,692 \pm 2 \%$ \\
\cline { 3 - 8 } & $0.026 \pm 7 \%$ & $0.140 \pm 3 \%$ & $1.019 \pm 1 \%$ & $8.051 \pm 2 \%$ & $63.75 \pm 1 \%$ & $513.8 \pm 1 \%$ \\
\hline \hline Shift-reduce parser & 33 & $0.005 \pm 9 \%$ & $0.039 \pm 2 \%$ & $0.307 \pm 1 \%$ & $2.439 \pm 1 \%$ & $19.73 \pm 2 \%$ & $157.2 \pm 1 \%$ \\
\hline Arithmetic operations & 33 & $0.005 \pm 9 \%$ & $0.038 \pm 3 \%$ & $0.294 \pm 1 \%$ & $2.336 \pm 1 \%$ & $18.85 \pm 1 \%$ & $150.8 \pm 1 \%$ \\
\hline
\end{tabular}

Table 3. Performance of Representative Programs with Sorted Data Structures Measured in Seconds

\begin{tabular}{|c|c|c|c|c|c|c|c|}
\hline \multirow{2}{*}{ Program } & \multirow{2}{*}{$\begin{array}{c}\text { Field } \\
\text { size }\end{array}$} & \multicolumn{6}{|c|}{ Data size } \\
\hline & & $2^{4}$ & $2^{5}$ & $2^{6}$ & $2^{7}$ & $2^{8}$ & $2^{9}$ \\
\hline \multirow{2}{*}{$\begin{array}{l}\text { Sorted linked list (DU) } \\
\text { (list building and traversal) }\end{array}$} & \multirow{2}{*}{81} & $0.466 \pm 1 \%$ & $1.908 \pm 1 \%$ & $7.750 \pm 1 \%$ & $31.24 \pm 1 \%$ & $125.5 \pm 1 \%$ & $565.5 \pm 1 \%$ \\
\hline & & $0.036 \pm 1 \%$ & $0.071 \pm 1 \%$ & $0.142 \pm 1 \%$ & $0.284 \pm 1 \%$ & $0.567 \pm 1 \%$ & $1.311 \pm 1 \%$ \\
\hline \multirow{3}{*}{$\begin{array}{l}\text { Sorted linked list (PU) } \\
\text { (list building, traversal, } \\
\text { and head node removal) }\end{array}$} & \multirow{3}{*}{81} & $1.464 \pm 1 \%$ & $9.956 \pm 1 \%$ & $85.51 \pm 2 \%$ & $918.6 \pm 2 \%$ & $9900 \pm 3 \%$ & $\mathrm{~N} / \mathrm{A}$ \\
\hline & & $0.051 \pm 1 \%$ & $0.149 \pm 2 \%$ & $0.613 \pm 2 \%$ & $5.285 \pm 2 \%$ & $45.93 \pm 2 \%$ & $\mathrm{~N} / \mathrm{A}$ \\
\hline & & $0.005 \pm 1 \%$ & $0.015 \pm 1 \%$ & $0.044 \pm 2 \%$ & $0.174 \pm 2 \%$ & $0.720 \pm 3 \%$ & $\mathrm{~N} / \mathrm{A}$ \\
\hline Mergesort without pointers & 81 & $0.053 \pm 5 \%$ & $0.121 \pm 5 \%$ & $0.271 \pm 5 \%$ & $0.625 \pm 4 \%$ & $1.453 \pm 4 \%$ & $3.124 \pm 4 \%$ \\
\hline Mergesort with pointers & 81 & $0.053 \pm 4 \%$ & $0.122 \pm 5 \%$ & $0.272 \pm 5 \%$ & $0.638 \pm 6 \%$ & $1.503 \pm 5 \%$ & $3.201 \pm 5 \%$ \\
\hline
\end{tabular}

additions. All linked list experiments contain two times for building and traversing the linked list, respectively, and we also report the time of the head node removal for sorted linked lists with pointer update. The tables also report the size of field elements in bits used to represent secret shared values. While all programs were written to work with 32-bit integers, most programs in these tables use statistically secure comparisons, which requires the length of the field elements to be increased by the statistical security parameter (which we set to 48). (The size of the field elements needs to be the size of the data plus one bit to ensure that all data values can be represented.)

The results tell us that working with linked lists (the first program in Table 2) in the secure computation framework is very efficient. That is, building a linked list that consists of thousands of elements takes a fraction of a second. Traversing a linked list is also rather quick, where going through a linked list of size $2^{11}$ takes about $1 \mathrm{~s}$ in our optimized program.

Performance of the sorted linked lists (the first two programs in Table 3) characterizes performance expected from different data structures where it is necessary to hide the place where a new node or data item is being inserted. As previously mentioned, there is no good reason to implement the PU variant of different data structures and it is provided here for sorted linked lists for illustration purposes only. The DU version of sorted linked list has the same list traversal time as the regular (unsorted) linked lists, and the reported time for sorted linked lists can be further optimized in the same way as it was done for regular linked lists. When we are building a sorted linked list via DU, each operation takes $O(n)$ time and thus the time to perform this operation for all $n$ elements of the input is $O\left(n^{2}\right)$. This quadratic performance is also observed empirically where increasing the size of the data set by a factor of 2 results in a four-time increase in the list building time (all insertion operations are performed sequentially). As far as the head node removal operation in a sorted linked list with PU goes, it consists of two pointer dereferences (i.e., using data and next fields) and one call to pfree, where the overhead of pfree was between $76.4 \%$ to $81.3 \%$ of that operation's time. In this particular experiment, each pointer stores $O(n)$ locations, 
which contributes to the complexity of both memory deallocation and pointer dereferencing, but the latter operation can be performed more efficiently.

If we next look at mergesort (the last two programs in Table 3), then we see that the variant that uses pointers to private data and makes a function call to a compare-and-exchange operation for each comparison and the variant with no pointers and corresponding function calls differ in their performance by a very small amount. The non-pointer version that performs less work is faster by $0.4-2.4 \%$.

Last, the performance of our shift-reduce parser (the second program in Table 2) is extremely fast and it almost entirely consists of the time it takes to evaluate the provided formula on private data (the last program in Table 2). That is, despite having a more complex functionality and employing pointer-based stack, the time to perform arithmetic operations only is almost the same as the time the parser takes. Also, adding pfree to the program does not effect the runtime (because the pointer stores a single address) and the times with memory deallocation are omitted from the table.

As far as memory consumption goes, the introduction of pointers to private data only marginally affects the amount of allocated memory for programs with pointers storing a single memory location (linked list, shift-reduce parser, and mergesort). The amount of memory needed to store and process sorted linked lists is quadratic in the data size and matches in its complexity list traversal. Removing a node from the list and calling pfree reduces the memory consumed by the data. While in general calls to pfree can increase memory consumption, in this case all pointers store the same lists of $O(n)$ locations and removing a node and merging the lists in pfree decreases the size of each list. In general, we can say that memory consumption is at most quadratic in the amount of data and user-declared variables in any program.

We note that all functionalities used for our experiments have alternative implementations using arrays. For linked lists, mergesort, and a shift-reduce parser, we expect array-based implementation to exhibit very similar performance to that based on pointers, because all pointers store a single location. For sorted linked lists, we expect array-based programs to have performance similar to our data update implementation (with the same asymptotic complexities). To confirm this finding, we evaluated performance of array-based sorted linked lists, the source code of which can be found in the full version [25] (Figure 6). Building the sorted list took about $20 \%$ less time using arrays for most data sizes, while list traversal was about $9 \%$ slower using arrays for most data sizes. Thus, both implementations exhibit comparable performance. Memory consumption is also similar in most programs, with the exception of array-based sorted list implementation that uses memory linear in the data size.

Performance of our pointer-based programs can also be compared to that of array-based implementations using another system or compiler. Sharemind [4] is a powerful system that supports a wide range of programs and, similar to PICCO, builds on (a different type of) information-theoretic secret sharing, which is hand-optimized to work with three computational parties. Despite similarities of the setup, Sharemind programs exhibit significantly different performance characteristics. In particular, the implementation is optimized for performing a large number of identical operations in a batch, while the cost of performing only a single operation is high (e.g., on the order of $100 \mathrm{~ms}$ for a single integer equality test [5]). As such, Sharemind programs will perform significantly worse (i.e., orders of magnitude slower) on our programs that perform sequential execution, such as unoptimized linked list traversal, the shift-reduce parser, and building a sorted linked list (mergesort is also slower as reported in Reference [26]). In the case of optimized linked list traversal, on the other hand, Sharemind implementations will still be slower for small data sets (such as $2^{5}$ ) but significantly faster for large data sets (up to two orders of magnitude faster for $2^{20}$ elements). 
All of this demonstrates that pointers have a great potential for their use in general-purpose programs evaluated over private data. Some pointer-based data structures can exhibit substantially slower performance in this framework than their public-data counterparts, and custom, internally built implementations for such data structures are recommended.

\section{CONCLUSIONS}

In this work, we introduce the first solution that incorporates support for pointers to private data into a general-purpose secure multi-party computation compiler. To maintain efficiency of pointerbased implementations, we distinguish between pointers with public addresses and pointers with private addresses and introduce the latter only when necessary. We provide an extensive evaluation of the impact of our design on various features of the programming language as well as evaluate performance of commonly used pointer-based data structures. Our analysis and empirical experiments indicate that the cost of using pointers to private data is minimal in many cases. Several pointer-based data structures retain their best-known complexities when they are used to store private data. Complexity of others (most notably balanced search trees) increases due to the use of private data flow, and custom, internally built implementations of oblivious data structures that work with sorted data are recommended. We hope that this work provides valuable insights into the use of various programming language features when developing programs for secure computation using a general-purpose compiler, as well as highlight benefits and limitations of pointer-based designs for SMC compiler developers.

\section{ACKNOWLEDGMENTS}

We thank Ethan Blanton for discussions at early stages of this work and anonymous reviewers for their valuable feedback.

\section{REFERENCES}

[1] GMP-The GNU Multiple Precision Arithmetic Library. Retrieved from http://gmplib.org.

[2] Mehrdad Aliasgari, Marina Blanton, Yihua Zhang, and Aaron Steele. 2013. Secure computation on floating point numbers. In Proceedings of the Network \& Distributed System Security Symposium (NDSS'13).

[3] Assaf Ben-David, Noam Nisan, and Benny Pinkas. 2008. FairplayMP: A system for secure multi-party computation. In Proceedings of the ACM Conference on Computer and Communications Security (CCS'08). 257-266.

[4] Dan Bogdanov, Sven Laur, and Jan Willemson. 2008. Sharemind: A framework for fast privacy-preserving computations. In Proceedings of the European Symposium on Research in Computer Security (ESORICS'08). 192-206.

[5] D. Bogdanov, M. Niitsoo, T. Toft, and J. Willemson. 2012. High-performance secure multi-party computation for data mining applications. Int. f. Info. Secur. 11, 6 (2012), 403-418.

[6] Ivan Damgård, Martin Geisler, Mikkel Krøigaard, and Jesper Buus Nielsen. 2009. Asynchronous multiparty computation: Theory and implementation. In Public Key Cryptography (PKC'09). 160-179.

[7] D. Demmler, G. Dessouky, F. Koushanfar, A.-R. Sadeghi, T. Schneider, and S. Zeitouni. 2015. Automated synthesis of optimized circuits for secure computation. In Proceedings of the ACM Conference on Computer and Communications Security (CCS'15). 1504-1517.

[8] D. Demmler, T. Schneider, and M. Zohner. 2015. ABY - A framework for efficient mixed-protocol secure two-party computation. In Proceedings of the Network and Distributed System Security Symposium (NDSS'15).

[9] Wilko Henecka, Ahmad-Reza Sadeghi, Thomas Schneider, and Immo Wehrenberg. 2010. TASTY: Tool for automating secure two-party computations. In Proceedings of the ACM Conference on Computer and Communications Security (CCS’10). 451-462.

[10] Andreas Holzer, Martin Franz, Stefan Katzenbeisser, and Helmut Veith. 2012. Secure two-party computations in ANSI C. In Proceedings of the ACM Conference on Computer and Communications Security (CCS'12). 772-783.

[11] Marcel Keller and Peter Scholl. 2014. Efficient, oblivious data structures for MPC. In Proceedings of the Annual International Conference on the Theory and Applications of Cryptology (ASIACRYPT'14). 506-525.

[12] A. Kiss and T. Schneider. 2016. Valiant's universal circuit is practical. In Proceedings of the Conference on Advances in Cryptology (EUROCRYPT'16). 699-728. 
[13] Benjamin Kreuter, Abhi Shelat, Benjamin Mood, and Kevin Butler. 2013. PCF: A portable circuit format for scalable two-party secure computation. In Proceedings of the USENIX Security Symposium. 321-336.

[14] Chang Liu, Yan Huang, Elaine Shi, Jonathan Katz, and Michael Hicks. 2014. Automating efficient RAM-model secure computation. In Proceedings of the IEEE Symposium on Security and Privacy. 623-638.

[15] Chang Liu, Xiao Shaun Wang, Kartik Nayak, Yan Huang, and Elaine Shi. 2015. ObliVM: A Programming framework for secure computation. In Proceedings of the IEEE Symposium on Security and Privacy.

[16] Dahlia Malkhi, Noam Nisan, Benny Pinkas, and Yaron Sella. 2004. Fairplay-Secure two-party computation system. In Proceedings of the USENIX Security Symposium.

[17] John Mitchell and Joe Zimmerman. 2014. Data-oblivious data structures. In Proceedings of the Symposium on Theoretical Aspects of Computer Science (STACS'14). 554-565.

[18] B. Mood, D. Gupta, H. Carter, K. Butler, and P. Traynor. 2016. Frigate: A Validated, extensible, and efficient compiler and interpreter for secure computation. In Proceedings of the IEEE European Symposium on Security and Privacy (EuroS\&P'16)

[19] Adi Shamir. 1979. How to share a secret. Commun. ACM 22, 11 (1979), 612-613.

[20] E. Songhori, S. Zeitouni, G. Dessouky, T. Schneider, A.-R. Sadeghi, and F. Koushanfar. 2016. GarbledCPU: A MIPS processor for secure computation in hardware. In Proceedings of the ACM Design Automation Conference (DAC'16).

[21] Ebrahim M. Songhori, Siam U. Hussain, Ahmad-Reza Sadeghi, Thomas Schneider, and Farinaz Koushanfar. 2015. TinyGarble: Highly compressed and scalable sequential garbled circuits. In Proceedings of the IEEE Symposium on Security and Privacy.

[22] Tomas Toft. 2011. Secure data structures based on multi-party computation. In Proceedings of the ACM Symposium on Priniciples of Distributed Computing (PODC'11). 291-292.

[23] Xiao Shaun Wang, Kartik Nayak, Chang Liu, T.-H. Chan, Elaine Shi, Emil Stefanov, and Yan Huang. 2014. Oblivious data structures. In Proceedings of the ACM Conference on Computer and Communications Security (CCS'14). 215-226.

[24] S. Zahur and D. Evans. 2013. Circuit structures for improving efficiency of security and privacy tools. In Proceedings of the IEEE Symposium on Security and Privacy. 493-507.

[25] Y. Zhang, M. Blanton, and G. Almashaqbeh. 2015. Implementing Support for Pointers to Private Data in a GeneralPurpose Secure Multi-Party Compiler. arXiv Report 1509.01763.

[26] Yihua Zhang, Aaron Steele, and Marina Blanton. 2013. PICCO: A general-purpose compiler for private distributed computation. In Proceedings of the ACM Conference on Computer and Communications Security (CCS'13). 813-826.

Received December 2016; revised August 2017; accepted October 2017 